\title{
Potential of Empirical Mode Decomposition for Hilbert Demodulation of Acoustic Emission Signals in Gearbox Diagnostics
}

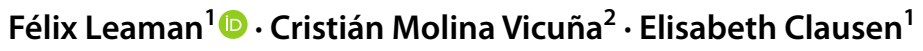

Received: 25 March 2021 / Revised: 6 July 2021 / Accepted: 1 September 2021 / Published online: 18 October 2021

(c) The Author(s) 2021

\begin{abstract}
Background The acoustic emission (AE) analysis has been used increasingly for gearbox diagnostics. Since AE signals are of non-linear, non-stationary and broadband nature, traditional signal processing techniques such as envelope spectrum must be carefully applied to avoid a wrong fault diagnosis. One signal processing technique that has been used to enhance the demodulation process for vibration signals is the empirical mode decomposition (EMD). Until now, the combination of both techniques has not yet been used to improve the fault diagnostics in gearboxes using AE signals.

Purpose In this research we explore the use of the EMD to improve the demodulation process of AE signals using the Hilbert transform and enhance the representation of a gear fault in the envelope spectrum.

Methods AE signals were measured on a planetary gearbox (PG) with a ring gear fault. A comparative signal analysis was conducted for the envelope spectra of the original AE signals and the obtained intrinsic mode functions (IMFs) considering three types of filters: highpass filter in the whole AE range, bandpass filter based on IMF spectra analysis and bandpass filter based on the fast kurtogram.

Results It is demonstrated how the results of the envelope spectrum analysis can be improved by the selection of the relevant frequency band of the IMF most affected by the fault. Moreover, not considering a complementary signal processing technique such as the EMD prior the calculation of the envelope of AE signals can lead to a wrong fault diagnosis in gearboxes. Conclusion The EMD has the potential to reveal frequency bands in AE signals that are most affected by a fault and improve the demodulation process of these signals. Further research shall focus on overcome issues of the EMD technique to enhance its application to AE signals.
\end{abstract}

Keywords Acoustic emission $\cdot$ Condition monitoring $\cdot$ Fault diagnostics $\cdot$ Empirical mode decomposition $\cdot$ Envelope analysis

\section{Introduction}

In recent years, the acoustic emission (AE) analysis has been used increasingly for fault diagnosis of gearboxes. Some investigations have shown the advantages of AE over conventional vibration analysis for several cases. Loutas et al. [23] evaluated several vibration and AE features to detect crack propagation in a parallel shaft gearbox. They concluded that AE features have better capabilities for tracking

Félix Leaman

fleaman@amt.rwth-aachen.de

1 Institute for Advanced Mining Technologies, RWTH Aachen University, Wüllnerstr. 2, 52064 Aachen, Germany

2 Laboratorio de Vibraciones Mecánicas, Universidad de Concepción, Concepción, Chile the damage progression, especially at earlier stages. Regionally linear behavior of some AE features was observed and gradient changes were associated with changes in the crack propagation rate. Tan et al. [30] compared the feasibility of using AE RMS value, vibration RMS value and iron concentration for monitoring of pitting in a parallel shaft gearbox. They concluded that the AE RMS value measured near the pinion had the best sensitivity overall. However, its good performance was decreased when the AE sensor was located on the bearing housing due to the change in the transmission path and addition of AE coming from other sources. Leaman et al. [18] analyzed vibration and AE signals measured on the outside of a ring gear from a full-size wind turbine gearbox. The comparison considered a non-faulty case and a faulty case with a small surface defect in one tooth from the ring gear. They conducted a spectral analysis of operational 
frequencies and concluded that the defect was better detected in the $\mathrm{AE}$ signals.

In gear transmissions, the asperity contacts between meshing teeth have been indicated as the main source of AE [31]. Thus, the presence of tooth damage can affect those asperity contacts and change the characteristics of the generated AE. For instance, results from Eftekharnejad and Mba [8] indicated a correlation between the AE RMS value and fault size of a tooth from a parallel shaft gearbox. They attributed this result to the higher number of surface protusions that increase the asperity contacts for bigger faults. Therefore, similar to the vibration analysis, a tooth defect can produce amplitude modulations in the AE signals with certain characteristic frequency. In the study from Novoa and Vicuña [26] they used envelope spectrum analysis of AE signals to detect modulations due to defects in sun and planet gears from a planetary gearbox (PG). In their approach, the envelope was obtained using the Hilbert transform applied direct on the AE signals. Their results showed that for sun gear defect and planet gear defect (external meshing only), the envelope spectra contained several harmonics of the component characteristic fault frequency, which was not the case for the non-faulty case. For the planet gear defect (internal meshing) they could not establish a difference with the non-faulty case. Other authors like Gao et al. [12] have also used a Hilbert transform to perform envelope analysis for gear fault diagnosis using AE signals.

Since AE signals are of nonlinear, non-stationary and broadband nature, the demodulation with the Hilbert transform may involve quadrature errors that can interfere with a correct fault diagnosis [27]. A well-known method to improve the calculation of the envelope consists of finding an optimal frequency band to filter the signal prior the demodulation process. In the literature there are several approaches to find this optimal demodulation band in order to increase the performance of gearbox fault diagnostics. Although most of them have been developed and tested for vibration signals, the approaches are still (at least) methodologically valid for AE signals. Antoni [1] defined the spectral kurtosis which is a tool to identify series of transients in the frequency domain that can be originated from faults. He proposed an estimator of the spectral kurtosis based on the short-time Fourier transform that helps to find the frequency band with the maximal kurtosis. In another paper [3], Antoni proposed another estimator, the fast kurtogram, based on 1/3 binary filter banks. Sawalhi et al. [28] proposed the use of the minimum entropy deconvolution to enhance the performance of the spectral kurtosis by minimizing the effect of the signal transmission path. McDonald et al. [25] proposed the maximum correlated kurtosis method that takes advantage of the periodic nature of faults to avoid high kurtosis values from random events. Other approaches have also applied the wavelet transform and wavelet packet decomposition to find the optimal demodulation band. Hemmati et al. [13] proposed the selection of a wavelet level corresponding to certain frequency band based on the maximization of the kurtosis to Shannon entropy ratio of the envelopes of the wavelet coefficients. Liu et al. [21] proposed an adaptive spectral kurtosis filtering based on Morlet wavelet. Zhang et al. [37] proposed a method based on the correlated kurtosis of envelope signal extracted from the wavelet packet decomposition. More recently, there have been approaches for selection the of optimal demodulation frequency band using evolutionary computing. Wang et al. [33] proposed a genetic algorithm to find the optimal bandpass filter that maximizes the signal-to-noise ratio of fault-related components such as characteristic fault frequencies. Kannan et al. [16] also proposed a genetic algorithm using a triangle series crossover and a novel fitness function based on the fault-related peaks to highest peaks ratio in the envelope spectrum. Some other approaches are based on the empirical mode decomposition (EMD) technique, which is presented in the next paragraph.

The EMD is a technique used to decompose complex signals into a collection of nearly orthogonal intrinsic mode functions (IMF). This decomposition reflects the local timescale characteristics of the signal. Moreover, these IMFs have well-behaved Hilbert transforms, from which instantaneous frequencies can be calculated as described by Huang et al. [15]. From another point of view, the EMD can be seen as an adaptive dyadic filter bank similar to the wavelet decompositions. In this sense, the first IMF corresponds to a high-pass filtered version of the signal, while other highorder IMFs correspond to band-pass filtered versions of the signal at decreasing center frequencies [11].

The procedure for extracting the IMFs is called sifting, which is an iterative procedure that in each iteration involves finding all local extrema in a signal and making two cubic spline interpolations. Since these operations are computationally expensive, the sifting process can last much time if the signals to analyze are measured with high sampling rates. Although this might not be a problem for vibration signals, the application of the EMD to AE signals can become problematic due to their very high sampling rates (commonly at least 20 times higher than for vibration). The EMD suffers from some drawbacks such as sensitivity to noise and mode mixing. These issues have been addressed by the use of an ensemble empirical mode decomposition (EEMD) proposed by $\mathrm{Wu}$ and Huang [34]. The EEMD consists of doing the EMD several times with different levels of artificially added noise and obtaining mean results for each IMF. As result, the mode mixing can be avoided. However, the calculation time will be considerably higher than for the simple EMD, making the application of the EEMD for AE signals extremely slow. More recently developed methods inspired by the EMD are the variational mode decomposition (VMD)

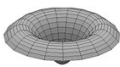


proposed by Dragomiretskiy and Zosso [7], and the independence-oriented VMD proposed by Li et al. [19]. The VMD is a non-recursive method that looks for an ensemble of modes with different center frequencies in a way that the combination of modes can reproduce the original signal. The independence-oriented VMD is an improvement that deals with the problem of finding the optimal number of modes based on their similarity. In the field of gearbox diagnostics, the EMD algorithm has been used mainly for the analysis of vibration signals, although there are some investigations that have addressed the application to AE signals.

Considering first the application to vibration signals, Feng et al. [9] estimated the envelope and instantaneous frequency of vibration signals using the energy separation algorithm applied on the obtained IMFs from an ensemble EMD. They conducted experiments in a two-stage PG for healthy, worn sun gear tooth and chipped sun gear tooth conditions. Their spectral analysis revealed several components at the sun gear fault characteristic frequency for the faulty cases. Loutridis [24] proposed an experimental law that relates the energy content of the different IMFs to tooth crack magnitude in a parallel shaft gearbox. In the work of Liu et al. [22] with vibration data from an automobile gearbox, they concluded that the use of EMD could produce better results for fault diagnosis than the continuous wavelet transform. A more advanced method that combines the advantages of the EMD and wavelet analysis is the empirical wavelet transform (EWT), which was successfully used for bearing fault diagnosis in the work from [6]. Feng at al. [10] used the VMD to decompose vibration signals from a PG and selected the most sensistive IMF based on a gear-fault related criteron. Subsequently, the carried out a joint amplitude-frequency Hilbert demodulation in order to calculate the envelope spectra of the signals. Teng at al. [32] compared the envelope spectra from bandpass-filtered versions of the original vibration signal with envelope spectra from the IMFs calculated with the EMD. With their experimental results, they concluded that the envelope spectra from the IMFs have better potential for gear fault detection. Chen et al. [5] proposed a resampling of the original vibration signal before the application of the EEMD to increase computational efficiency. After the decomposition, they selected the IMF with highest kurtosis to implement a Hilbert square demodulation and analyze faulty components in the envelope spectrum. Zhang et al. [38] used a genetically optimized VMD to obtain the IMFs of vibration signals from rolling bearings. They proposed to use the two IMFs with maximum kurtosis to reconstruct a signal, from which the envelope spectrum is calculated.

The number of investigations concerning the application of the EMD for gearbox diagnostics using AE signals is much lower than for vibration. Among those investigations, $\mathrm{Li}$ and $\mathrm{He}[20]$ proposed a fused feature calculated from the IMFs of AE signals to detect a cracked tooth in a splittorque gearbox. The feature consists of typical features such as RMS value, crest factor, AE burst count, peak value and burst duration. Yoon and $\mathrm{He}$ [35] investigated the use of the EMD to detect different faults (sun, planet and ring gear) in a PG with AE signals. Typical features were calculated from the IMFs of various input signals: raw, energy operator, narrow band, envelope, instantaneous frequency and the Welch's signal. The results showed that, depending of the fault, only certain features could separate effectively the healthy and faulty cases. Kedadouche et al. [17] applied the EMD to AE signals and calculated statistical features from the obtained IMFs. They validated their methodology with experiments from a bearing test bench with a crack in the outer ring.

As shown, there are few investigations conducted in the field of gearbox diagnostics considering the application of EMD to AE signals. These investigations have focused mainly on calculating statistical features from the obtained IMFs. However, to the best knowledge of the authors, the EMD has not been yet used together with the envelope analysis in AE signals to improve the gearbox diagnostics. The combination of both techniques has achieved good results for the vibration analysis, but its use for the $\mathrm{AE}$ analysis is still barely studied. Therefore, in this paper we present a case study, in which the application of both techniques is experimentally tested. The case study consists of a PG with a localized fault in its ring gear. The obtained results demonstrate the very good potential of the approach and open new research challenges for future investigations.

Accordingly, the main novelty of this article is to reveal the potential of the well-known EMD technique for improving the demodulation of $\mathrm{AE}$ signals in the field of gearbox diagnostics. The proposed approach establishes the foundations for the development of new diagnostic methods based on the measurement of AE instead of conventional vibration, offering possibilities for much earlier fault detection. Drawbacks and further improvements for the proposed approach are also discussed.

\section{Theoretical Background}

\section{Envelope Analysis}

The envelope analysis has been established as a powerful technique to detect gearbox faults through the study of signal amplitude modulations. Since a defect in rotating components such as gears and bearings will produce an amplitude modulation with the characteristic frequency at which the defect enters in contact with another component, the spectral analysis of the envelope can provide clear signs of the fault and its location. The performance of the envelope analysis can be enhanced by 
the selection of an appropriate frequency band in which the amplitude modulations are to be analyzed (optimal demodulation band). As described in the previous section, in the field of gearbox diagnostics approaches such as spectral kurtosis and genetic algorithms have been proposed for selection of the appropriate demodulation band.

The most straightforward way to demodulate a signal is with a low-pass filter. The filter is applied on a half-wave or full-wave rectified version of the original signal to obtain the amplitude envelope. The cut-off frequency of the filter has a major impact on the result. High cut-off frequencies can produce an envelope with ripples, while low cut-off frequencies provide low sensitivity to sudden amplitude changes. Another method to obtain the amplitude envelope is based on the Hilbert transform. Consider an arbitrary amplitude and frequency modulated signal $x(t)$. The Hilbert transform of the signal $x(t)$ is defined in Eq. (1) as follows:

$\mathcal{H}[x(t)]=x(t) * \frac{1}{\pi t}=\frac{1}{\pi} \int_{-\infty}^{\infty} \frac{x(\tau)}{t-\tau} d \tau$

Then, the analytic signal $x_{a}(t)$ of the original signal $x(t)$ is defined as follows in Eq. (2):

$x_{a}(t)=x(t)+i \mathcal{H}[x(t)]=r(t) e^{i \theta(t)}$

In Eq. (2) the analytic signal is presented in its rectangular and polar form, where $r(t)$ is its modulus and $\theta(t)$ its phase. Consider now that the signal $x(t)$ can be expressed generically as shown in Eq. (3):

$x(t)=a(t) \cdot \cos (\phi(t))$

where $a(t)$ is its amplitude envelope and $\omega(t)=\phi^{\prime}(t)$ its instantaneous frequency. The quadrature signal $x_{q}(t)$ of the original signal $x(t)$, i.e. a signal that has a $90^{\circ}$ phase shift with respect to $x(t)$, can be expressed as shown in Eq. (4): $x_{q}(t)=a(t) \cdot \sin (\phi(t))$

Then, if the quadrature signal $x_{q}(t)$ is equal to the Hilbert transform of $x(t)$, it can be demonstrated that $r(t)=a(t)$ and $\theta^{\prime}(t)=\omega(t)$. In general $\mathcal{H}[x(t)]$ and $x_{q}(t)$ are not equal, thus in the calculation of the amplitude envelope with this method there is an estimation error present. Therefore, $r(t)$ can only be considered as an approximation of the real amplitude envelope $a(t)$ [27]. A more realistic condition can be satisfied when the signal $x(t)$ is a narrowband signal, i.e. a signal with most of its energy centered around a single carrier frequency. In this case the analytic signal can be effectively used to extract the amplitude envelope of $x(t)$ [29].

\section{Empirical Mode Decomposition}

Let $x(t)$ be the signal under analysis. The IMFs of $x(t)$ are obtained iteratively using the sifting process illustrated in Fig. 1. First, the upper and lower envelopes of $x(t)$ are calculated by finding all the local extrema and connecting them with cubic splines. Then, the mean envelope is calculated by taking the average of the two aforementioned envelopes (see Fig. 1a). Finally, the mean envelope is subtracted from $x(t)$ and a signal $h_{11}(t)$ is obtained (see Fig. 1b). This process is repeated several times using the signal $h_{1 k}(t)$ obtained in the iteration $k$ as input signal for the sifting iteration $k+1$. When a stopping criterion is achieved in the iteration $n$, the signal $h_{1 n}(t)$ is designated $c_{1}(t)$ as the first IMF from $x(t)$. The difference between the signal $x(t)$ and $c_{1}(t)$ is called the residual $r_{1}(t)$. To obtain further IMFs the sifting process must be applied again to the residual from the last sifting. Therefore, to obtain the IMF $c_{j+1}(t)$ the sifting process must be applied to the residual $r_{j}(t)$. The residual is no longer

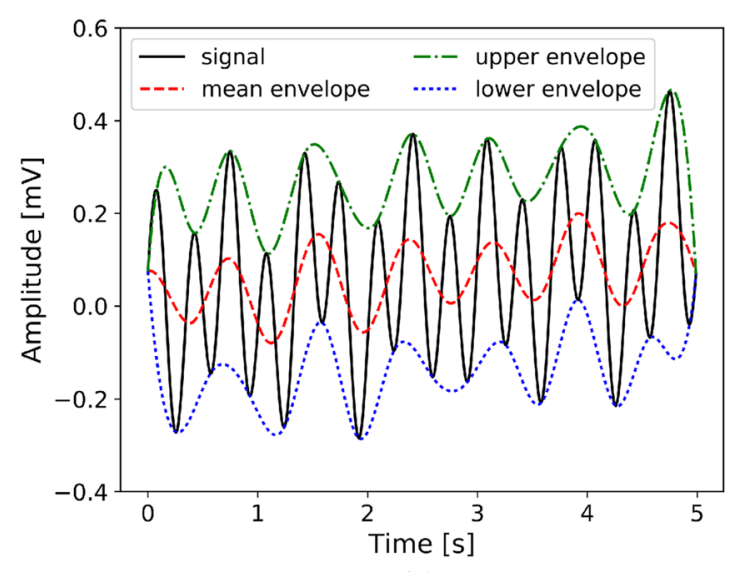

(a)

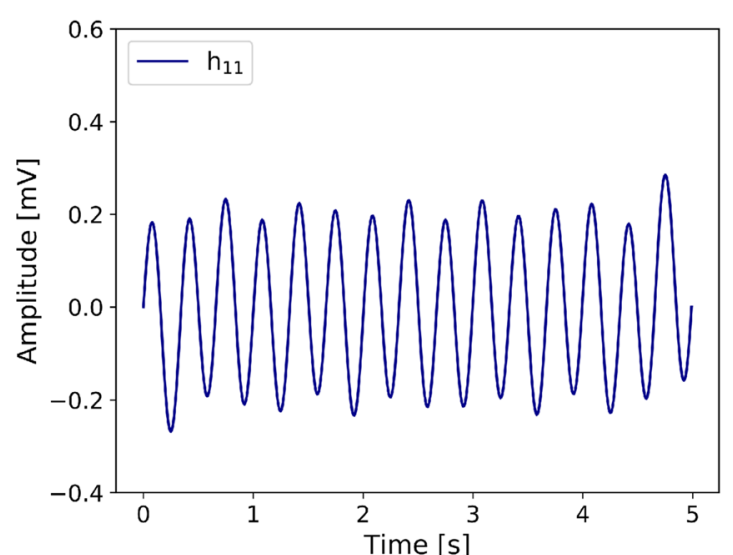

(b)

Fig. 1 Sifting process. In a the mean envelope calculation for the signal $h_{j k}(t)$ and in $\mathbf{b}$ the difference between the signal and its mean envelope to obtain $h_{j k+1}(t)$ 
useful, i.e. no more meaningful IMFs can be obtained from it, when it contains only a sine wave or no more oscillations.

The stopping criterion for the sifting process used in this study is known as the $s$-number criterion: when the number of relative extrema and zero-crossing of the signal are equal or differs at most by one for minimum $s$ iterations the sifting is stopped.

\section{Experimental Procedure}

AE signals were measured on a PG test bench. The ring gear outside diameter was $144 \mathrm{~mm}$. The gearbox had the following number of teeth: $Z_{s}=18$ for the sun gear, $Z_{r}=72$ for the ring gear and $Z_{p}=26$ for each of the $N_{p}=3$ planet gears. The speed reduction ratio was 1:5. The input (high speed) shaft was connected to an $22 \mathrm{~kW}$ electric motor and the output (low speed) shaft, to a generator. Both machines were connected to variable-frequency drives in order to adjust rotational speed (motor) and load (generator). A genearal overview of the test bench is shown in Fig. 2a. An AE sensor VS375-M was fixed under the gearbox case using a magnetic holder as shown in Fig. 2b. This sensor offers a good balance for $\mathrm{AE}$ measurements in the standard frequency range $(100-400 \mathrm{kHz})$ and in the high-frequency range $(>400 \mathrm{kHz})$. Besides, the sensor is not appropriate for AE measurements under $20 \mathrm{kHz}$. The surface under the sensor was previously polished and copper paste was applied between sensor and surface to improve the acoustic coupling.

The AE signals were measured in eight measurement campaigns (MCs), from which seven consisted of approximately $75 \mathrm{~min}$ of continuous operation and one of approximately $120 \mathrm{~min}$. For all MCs the AE signals were measured using an input rotational speed of 1300 RPM and a torque of $112 \mathrm{Nm}$ in the output shaft. Thus, the sun gear rotating frequency was $f_{s}=21.67 \mathrm{~Hz}$ and the carrier rotating frequency was $f_{c}=4.33 \mathrm{~Hz}$. For all measurements a sampling frequency of $1 \mathrm{MHz}$, an acquisition time of $5 \mathrm{~s}$ for the signals and a gain in the pre-amplifier of $37 \mathrm{~dB}$ were used. To avoid the influence of the temperature on the AE signals during the start-up of the gearbox in each MC, the signals used for the analysis were measured after that the gearbox case reached an approximately constant temperature This was accomplished after approximately $60 \mathrm{~min}$ in each MC. By this point the temperature of the gearbox case increased no more than $0.2^{\circ} \mathrm{C}$ in over two minutes.

Three damage conditions were considered for the analysis: a non-faulty condition, an initial faulty condition and a developed faulty condition. The AE signals of the non-faulty condition were obtained from the 1st MC. After that, the gearbox was disassembled to introduce a seeded fault and was once again assembled. A localized fault was produced in one tooth of the gear ring using a grinding tool as shown in Fig. 3. This kind of fault can be considered as a worn tooth. Then, the AE signals of the initial faulty condition were obtained from the 2nd MC. The AE signals of the developed faulty condition were obtained from the 9th MC. During this eight MCs the gearbox was never disassembled nor the AE sensor. Summing the MCs, the gearbox accumulated a total of $9.5 \mathrm{~h}$ of operation from the introduction of the fault until the signals used for the analysis of the developed faulty condition were measured. A posterior inspection revealed no

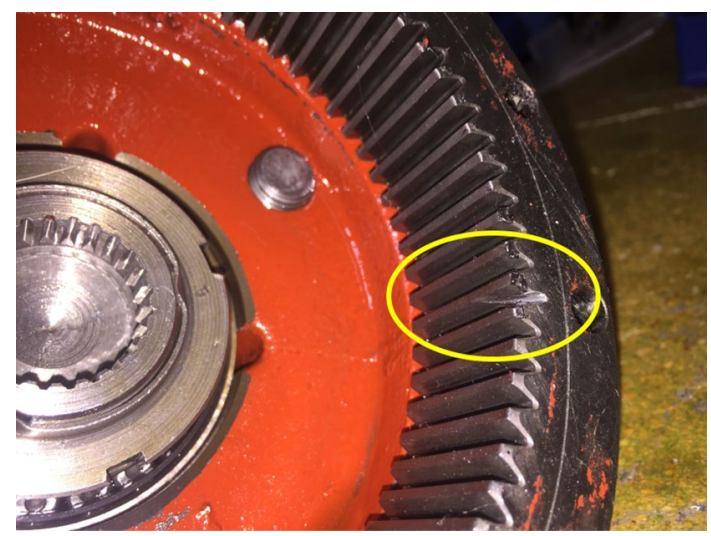

Fig. 3 Seeded Fault in a Tooth from the Ring Gear
Fig. 2 PG test bench. In a a general overview and in $\mathbf{b}$ the AE sensor

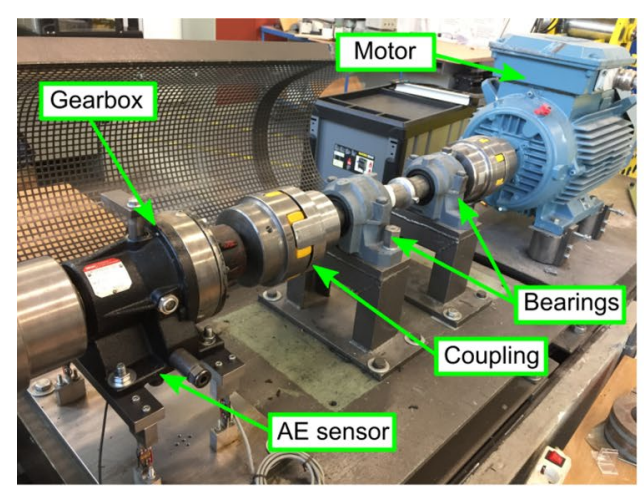

(a)

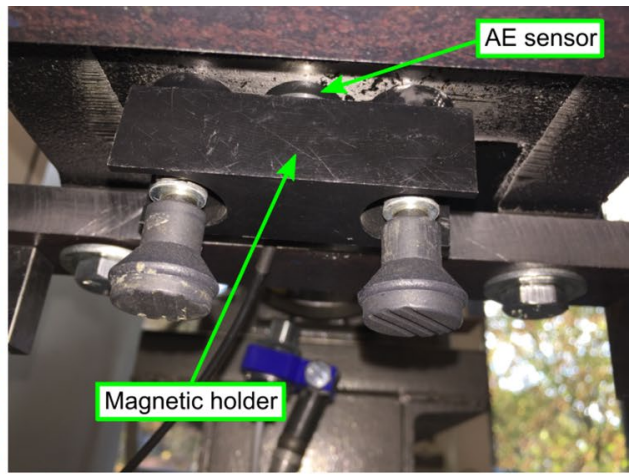

(b) 
visual growth of the fault, although damage progression at a microscopic level or tooth weakening cannot be discarded.

\section{AE Signal Analysis}

The analysis of the AE signals was based on spectral analysis of the envelopes. As first analysis, a high-pass filter was applied to the raw signals considering a cutoff frequency that preserves the main frequency range in which the $\mathrm{AE}$ phenomenon takes place and that can be measured by the $\mathrm{AE}$ sensor $(<20 \mathrm{kHz})$. The type of filter corresponds to a IIR Butterworth high-pass filter of order 3 . Then, the amplitude envelopes were obtained using the Hilbert transform with the analytic signal and the frequency spectra were obtained using the Fourier transform. Finally, the magnitudes of the fault characteristic frequency and its harmonics were obtained. For each of the three damage conditions the average spectrum of three consecutive signals was considered. For a localized fault in the ring gear, the fundamental frequency at which the planet gears interact with the fault is $N_{p} f_{c}$. However, this assumption is valid only when the interactions among the fault and all planet gears are very similar. Due to effects such as gearbox assembly, shaft alignment and surface characteristics of the planet gears the aforementioned condition rarely takes place, especially in the high-frequency range of $\mathrm{AE}$. A more general assumption is that the fundamental frequency for a localized ring gear fault is simply $f_{c}$.

As second analysis, the Fourier spectra of the IMFs were analyzed in order to find which IMF and associated frequency bands are most affected by the fault. This frequency band is selected visually by comparing the different frequency spectra of the IMF for different fault conditions. Once the appropriate IMF and frequency band were selected, a band-pass filter was applied to the original signal and to the IMF to isolate the frequency range most affected by the fault. The type of filter corresponds to a IIR Butterworth band-pass filter of order 3 . Then, the procedure described in the previous paragraph was repeated, i.e. obtaining the amplitude envelopes with the Hilbert transform, obtaining the Fourier spectra and evaluating the magnitudes of fault related components. To provide comparison, the spectra of the IMFs with only a $20 \mathrm{kHz}$ high-pass filter (instead of the band-pass filter) were also analyzed.

As third analysis, the fast kurtogram is analyzed as tool for selection of the relevant frequency band more suited to calculate the envelope spectra. In this case, the selected frequency band will be the band in which the bandpass filtered signal reaches a maximum kurtosis. The fast kurtogram algorithm was proposed by Antoni [2] and is based on an arborescent multirate filter-bank structure, where the frequency range is decomposed into $k$ levels. For the calculations made in this study a value $k=4$ was selected, which generates frequency band of bandwidth $31250 \mathrm{~Hz}$ for the deepest level. A main point of analysis is to evaluate if the frequency band that generates a maximum kurtosis is the same band as the one selected visually based on the fault condition. Fig. 4 provides a schematic overview of the analysis methodology.

To perform a quantifiable comparison and determine in which case the fault can be detected better, a mean-peak ratio (MPR) calculation was carried out [33]. The MPR was used to measure the magnitude of fault related components relative to each spectrum. The MPR is defined in Eqs. (5) and (6) as follows:

MPR $=20 \log _{10} \frac{\sum_{i=1}^{N_{h}}\left(P_{i}-A_{s}\right)}{A_{s}}$

$A_{s}=\frac{\sum_{k=a}^{b} S_{k}}{b-a}$

In Eqs. (5) and (6) $N_{h}$ indicates the number of harmonics of the frequency of interest, $P_{i}$ is the magnitude of the $i$ th harmonic of the frequency of interest, $A_{s}$ is the average value of the spectrum in the range from frequency $a$ to frequency $b$ and $S_{k}$ is the magnitude of the $k$ th spectral component in the range. Within this work, the fundamental frequency of interest is the ring gear fault frequency $f_{f, r}=f_{c}=4.33 \mathrm{~Hz}$ and the number of harmonics to analyze was set to $N_{h}=10$. Accordingly, the values of $a$ and $b$ were set to $3 \mathrm{~Hz}$ and 45 $\mathrm{Hz}$, respectively.

\section{Results}

\section{Original Signals}

Figure 5a-c show the envelope spectra of the AE signals filtered with a $20 \mathrm{kHz}$ high-pass filter (to consider the whole $\mathrm{AE}$ range measured by the sensor) for the non-faulty, initial faulty and developed faulty conditions, respectively. In the three figures there are components at $f_{c}=4.33 \mathrm{~Hz}$ and $f_{s}$ $=5 f_{c}=21.67 \mathrm{~Hz}$, which are considered normal since they

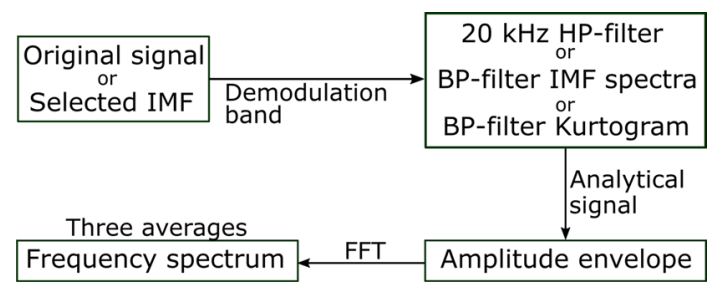

Fig. 4 Overview of the analysis methodology

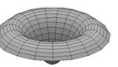




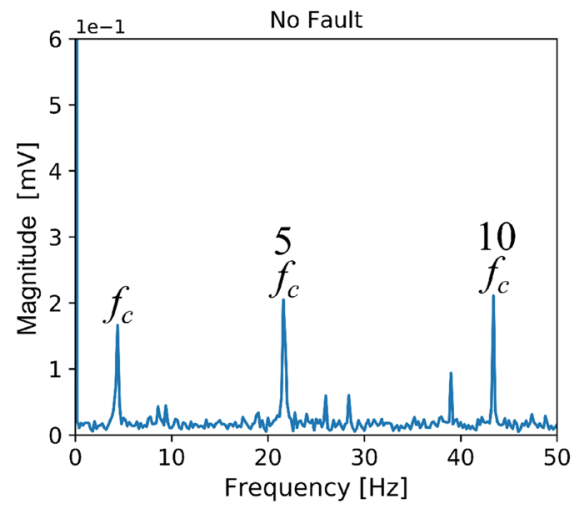

(a)

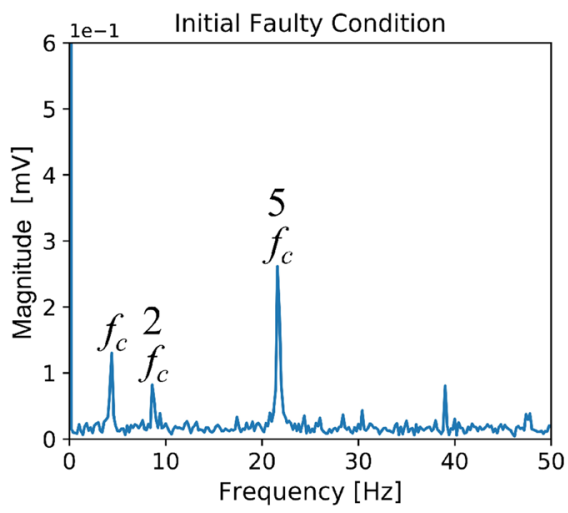

(b)

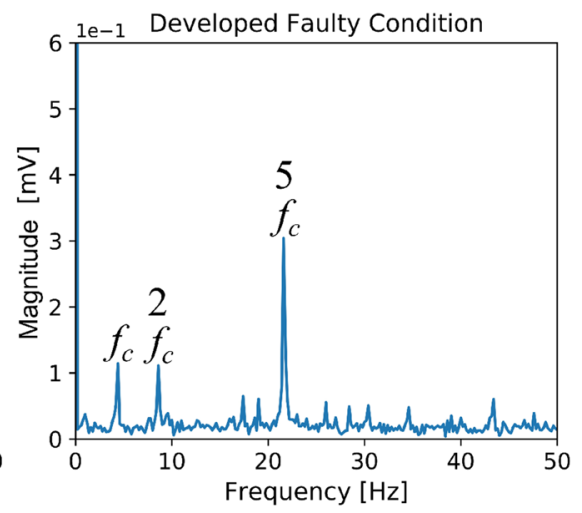

(c)

Fig. 5 Envelope spectra from the AE signals filtered with a $20 \mathrm{kHz}$ high-pass filter for the a non-faulty condition, $\mathbf{b}$ initial faulty condition and $\mathbf{c}$ developed faulty condition

are generated at each revolution of the carrier and sun gear, respectively. Since it was considered that a localized fault in the ring gear has a fundamental frequency $f_{f, r}=f_{c}$, it was expected that for the faulty cases multiple harmonics of 4.33 $\mathrm{Hz}$ should be visible. In Fig. 5a there is a high-magnitude component at $43.34 \mathrm{~Hz}$, which could be more related to the second harmonic of $f_{s}$ rather than a high-order harmonic of $f_{c}$ (although its frequency corresponds to $10 f_{c}$ ). This component decreases its magnitude in Fig. $5 \mathrm{~b}$ and c, which can be attributed to the new mounting condition after that the gearbox was disassembled and re-assembled to introduce the seeded fault. Focusing on fault-related components, a second harmonic of $f_{c}$ becomes visible for the initial faulty condition. However, when Fig. 5a and b are compared, there is no clear evidence of the fault since both figures show similar number of harmonics of $f_{c}$ with similar magnitudes. Similarly, when Fig. 5b and c are compared, there is also no clear evidence of fault development, i.e. the spectra do not show clear magnitude increases of fault-related components after the $9.5 \mathrm{~h}$ of operation.

In a further analysis, the frequency band between 95 and $140 \mathrm{kHz}$ was selected to conduct the demodulation of the $\mathrm{AE}$ signals. This particular frequency band was selected based on the upcoming analysis of the IMFs and is used here for comparison purposes. The results are shown in Fig. 6, where only minor changes in comparison with the results of using the whole AE frequency range are observed.

\section{Calculated IMFs}

The first five IMFs of the AE signals were obtained using the EMD with the $s$-number convergence criterion. An $s$-number of 2 was selected for all calculations. Fig. 7 shows the spectra of the three first IMFs for the non-faulty, initial and developed faulty conditions. For the first IMF (see Fig. 7a-c) there is a corresponding magnitude increase in the range

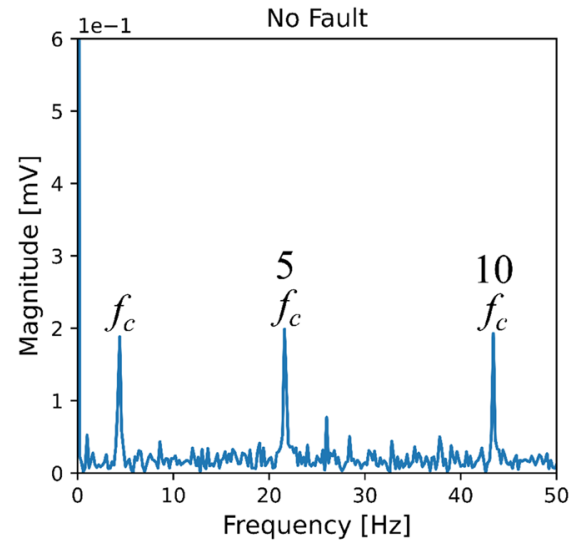

(a)

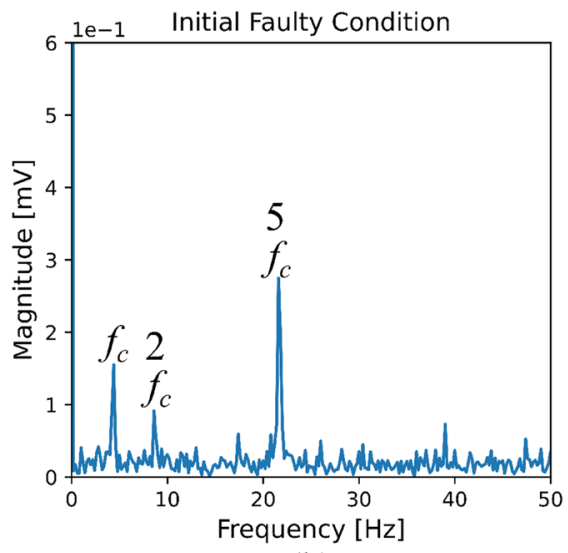

(b)

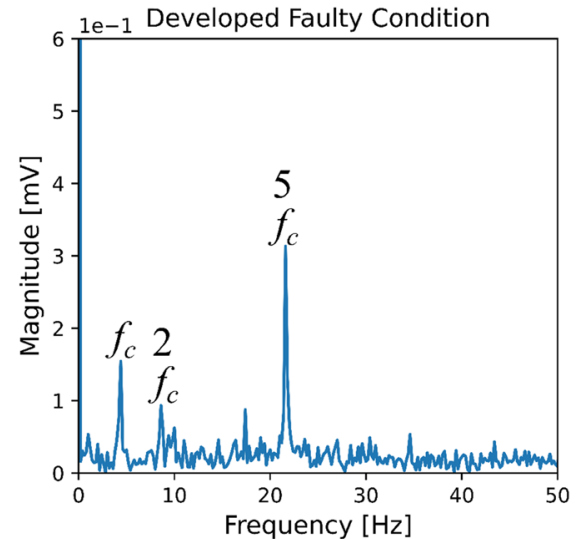

(c)

Fig. 6 Envelope spectra from the AE signals filtered with a $95-140 \mathrm{kHz}$ band-pass filter for the a non-faulty condition, $\mathbf{b}$ initial faulty condition and $\mathbf{c}$ developed faulty condition 


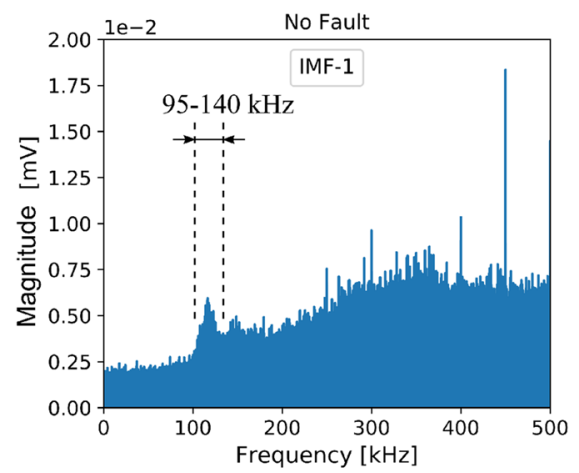

(a)

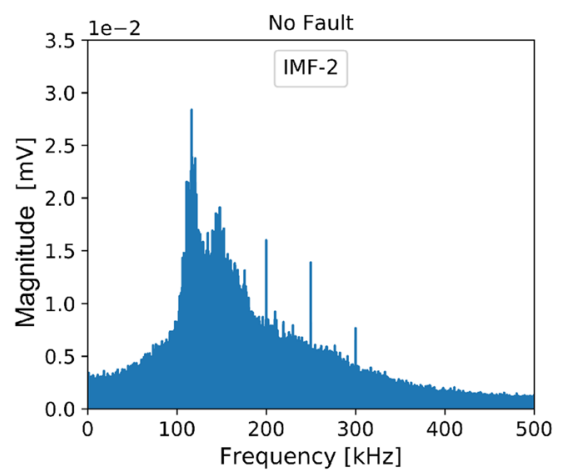

(d)

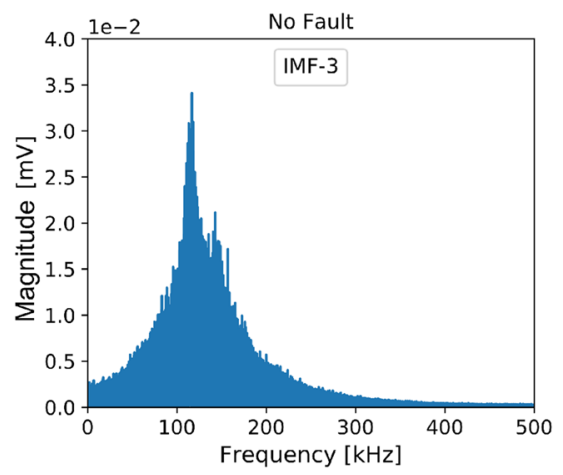

(g)

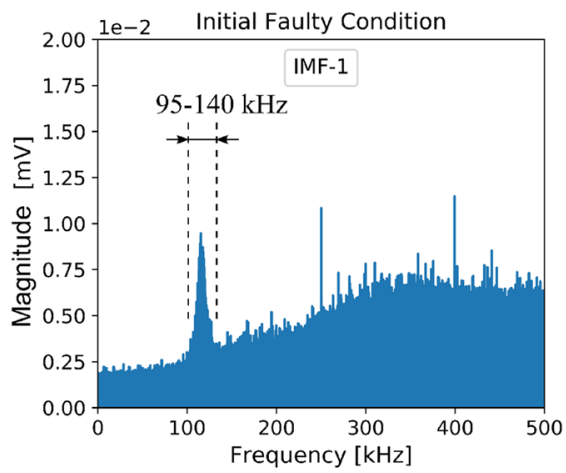

(b)

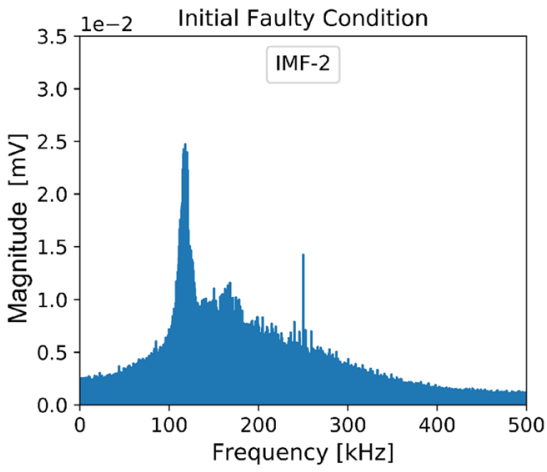

(e)

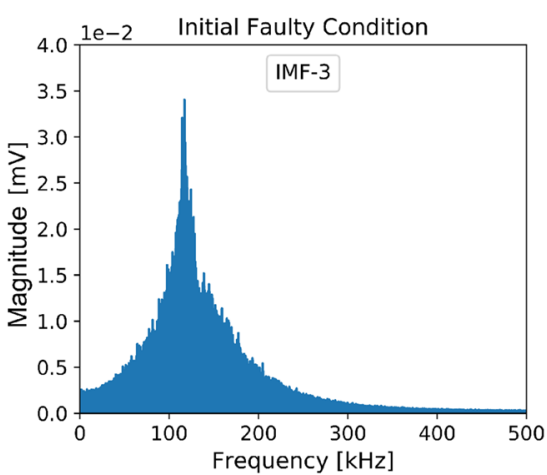

(h)

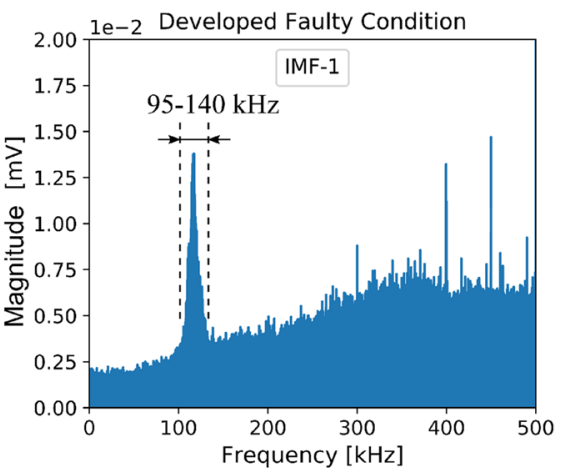

(c)

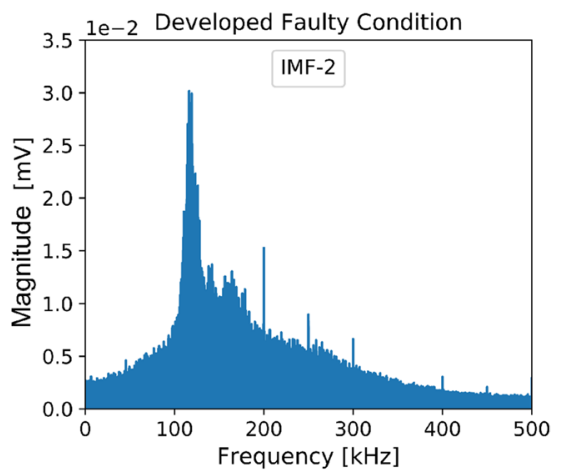

(f)

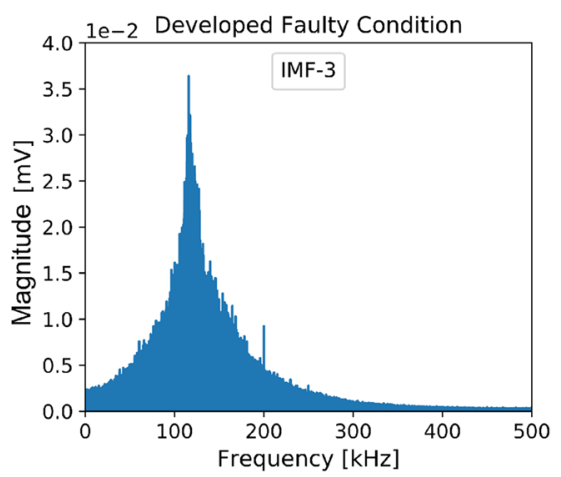

(i)

Fig. 7 Spectra of: a IMF-1 without fault, b IMF-1 with initial fault, c IMF-1 with developed fault, d IMF-2 without fault, e IMF-2 with initial fault, f IMF-2 with developed fault, $\mathbf{g}$ IMF-3 without fault, $\mathbf{h}$ IMF-3 with initial fault and i IMF-3 with developed fault

between 95 and $140 \mathrm{kHz}$ (highlighted zone), while the other frequency ranges remain approximately unchanged. For the second IMF (see Fig. 7d-f) there are magnitude changes in the range between 90 and $180 \mathrm{kHz}$, but they do not show a corresponding trend with the fault. For the third IMF (see Fig. $7 \mathrm{~g}-\mathrm{i}$ ) there are only minor magnitude changes among the three spectra. This last observation applies also to the fourth and fifth IMFs, which are not displayed in the figure. Based on this observation, the first IMF is considered primarily as the most suited for the analysis.

As first approach, the envelope spectra from the first IMF using the whole AE frequency range above $20 \mathrm{kHz}$ were analyzed. The results in Fig. 8 show that the highmagnitude component at $21.67 \mathrm{~Hz}$ is no longer visible for the non-faulty condition. In Fig. 8a a few harmonics of $f_{c}$ are visible above the spectral noise. For the initial faulty condition in Fig. $8 \mathrm{~b}$ several harmonics of $f_{c}$ are visible, while others have magnitudes only slightly higher than the spectral noise. For the developed faulty condition in Fig. 8c all harmonics within the frequency range of analysis are clearly visible. Most of them have higher magnitudes than the corresponding harmonics shown for the initial faulty condition, which can be a sign of damage progression in small scale. 


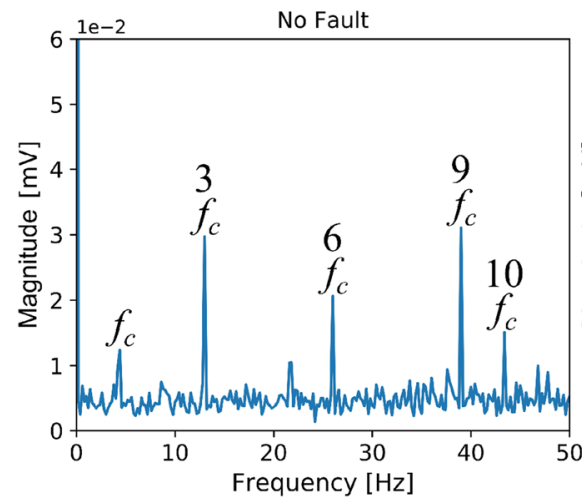

(a)

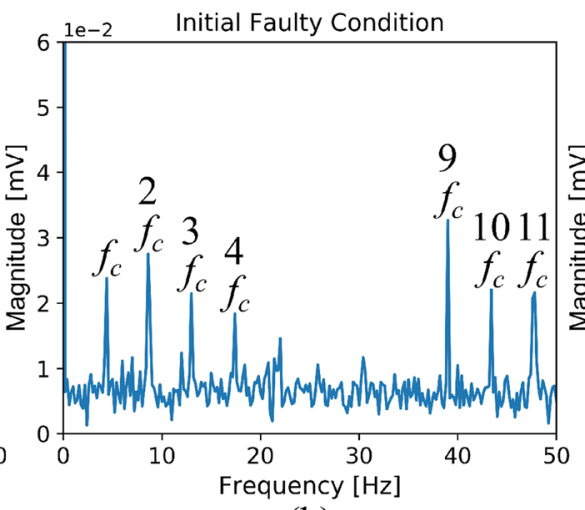

(b)

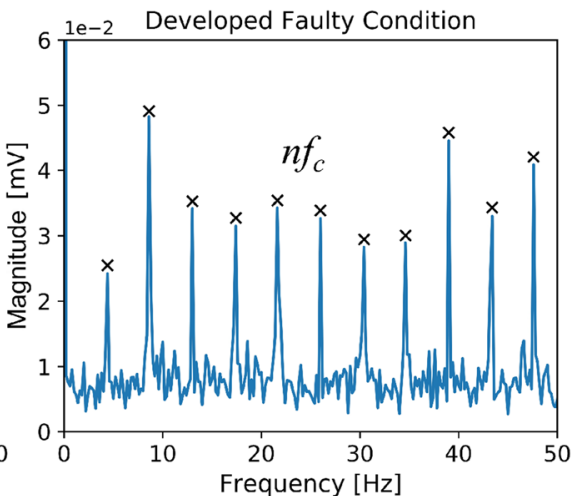

(c)

Fig. 8 Envelope spectra from the IMF-1 filtered with a $20 \mathrm{kHz}$ high-pass filter for the a non-faulty condition, $\mathbf{b}$ initial faulty condition and $\mathbf{c}$ developed faulty condition

In the subsequent approach, the envelope spectra from the first IMF were obtained using the frequency range between 95 and $140 \mathrm{kHz}$ as demodulation band. This frequency range was selected visually according to the magnitude increases from Fig. 7 as the frequency band most affected by the fault. Fig. 9 shows the respective obtained envelope spectra. The behavior of the harmonics is more clear, since they show an increasing tendency in their magnitudes. Firstly, for the non-faulty condition in Fig. 9a only the component at the fundamental frequency $f_{c}$ is visible above the spectral noise. Then, for the initial faulty condition in Fig. 9b several harmonics of $f_{c}$ are visible above the spectral noise. Finally, for the developed faulty condition in Fig. 9c all harmonics increase their magnitudes and also new harmonics became visible above the spectral noise.

In order to have more insights regarding the behavior of the other IMFs, the conducted analysis for the IMF-1 was repeated for the IMF-2 and IMF-3. These results are shown in Figs. 10, 11, 12, 13. In general terms, there are no major differences between the obtained spectra for IMF-2 using the high-pass filter (see Fig. 10) or the bandpass filter (see Fig. 11), and between the obtained spectra for IMF-3 using the high-pass filter (see Fig. 12) or the band-pass filter (see Fig. 13). Regarding the results for the IMF-2, there is no clear increase in the number or magnitude of the harmonics from $f_{c}$ as the fault develops. While the harmonic at $5 f_{c}=21.67 \mathrm{~Hz}$ increases its magnitude slightly, the magnitudes of the harmonics at $9 f_{c}=39 \mathrm{~Hz}$ and $10 f_{c}=43.33 \mathrm{~Hz}$ decrease. Regarding the results for the IMF-3, there is also no clear increase in the number or magnitude of the harmonics from $f_{c}$ as the fault develops. While the harmonic at $5 f_{c}=21.67 \mathrm{~Hz}$ increases its magnitude, the magnitudes of the harmonics at $f_{c}=4.33 \mathrm{~Hz}$ and $10 f_{c}=43.33 \mathrm{~Hz}$ decrease.

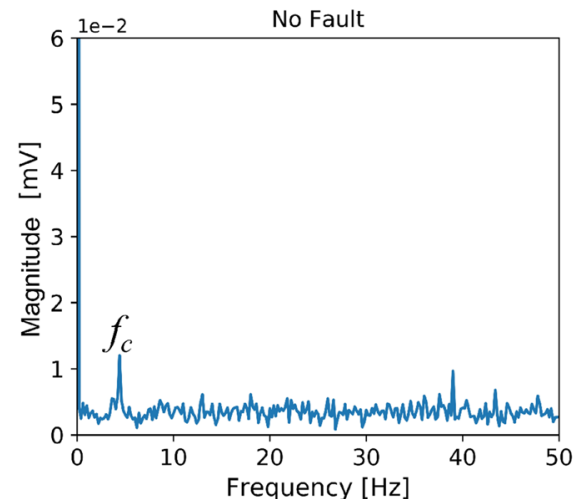

(a)

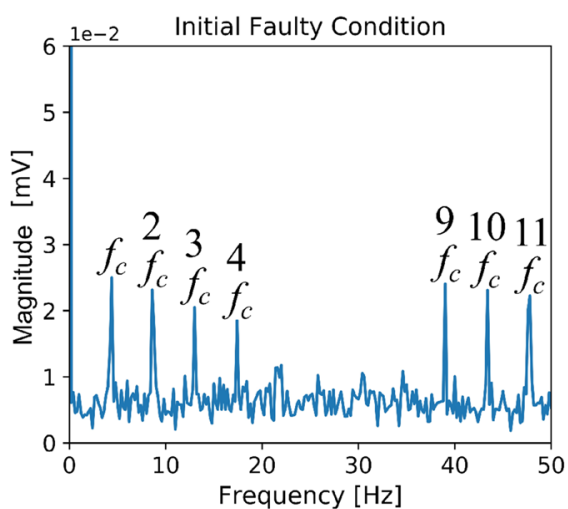

(b)

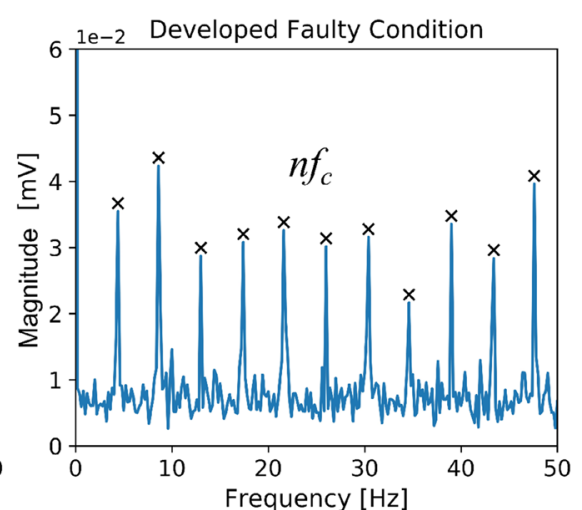

(c)

Fig. 9 Envelope spectra from the IMF-1 filtered with a $95-140 \mathrm{kHz}$ band-pass filter for the a non-faulty condition, b initial faulty condition and c developed faulty condition 


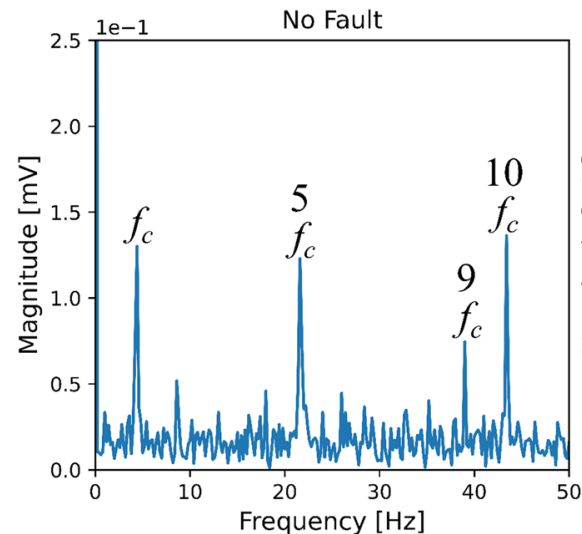

(a)

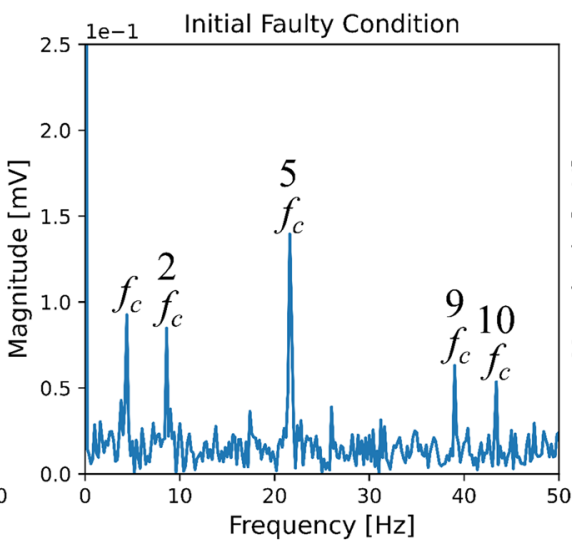

(b)

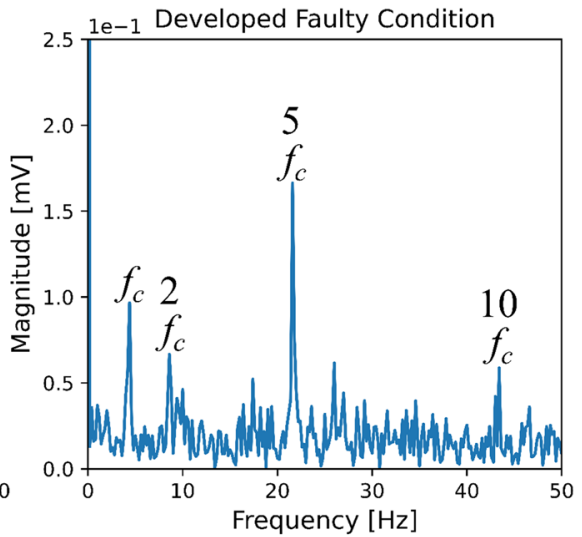

(c)

Fig. 10 Envelope spectra from the IMF-2 filtered with a $20 \mathrm{kHz}$ high-pass filter for the a non-faulty condition, b initial faulty condition and $\mathbf{c}$ developed faulty condition

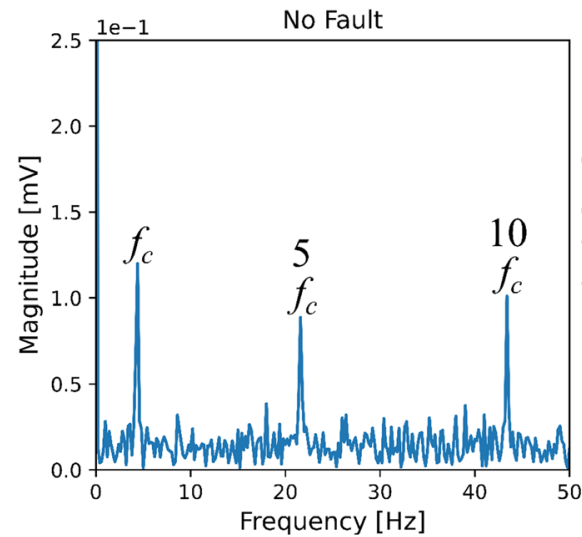

(a)

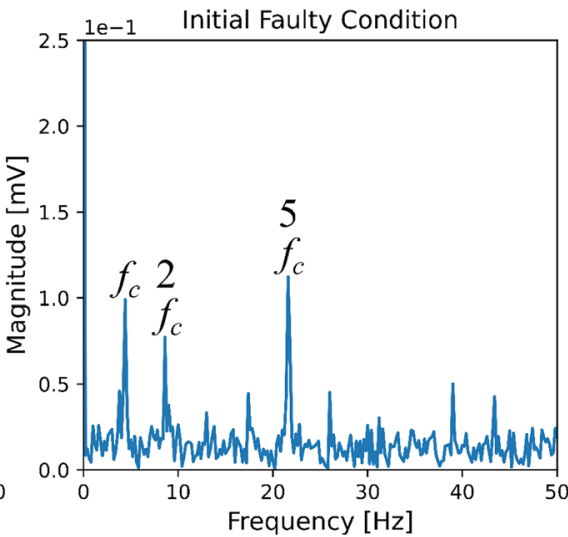

(b)

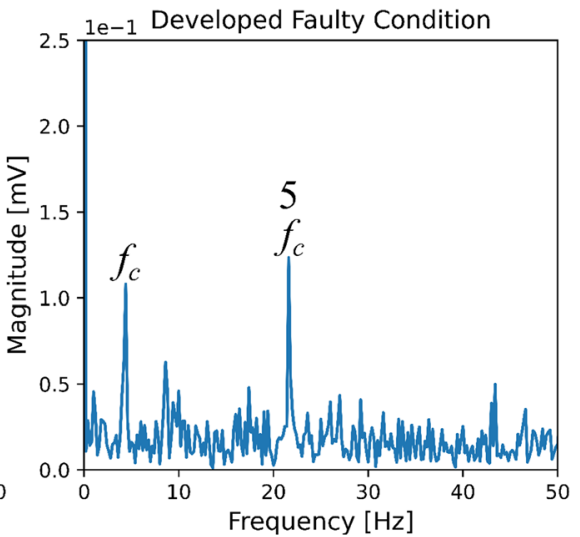

(c)

Fig. 11 Envelope spectra from the IMF-2 filtered with a $95-140 \mathrm{kHz}$ band-pass filter for the a non-faulty condition, $\mathbf{b}$ initial faulty condition and c developed faulty condition

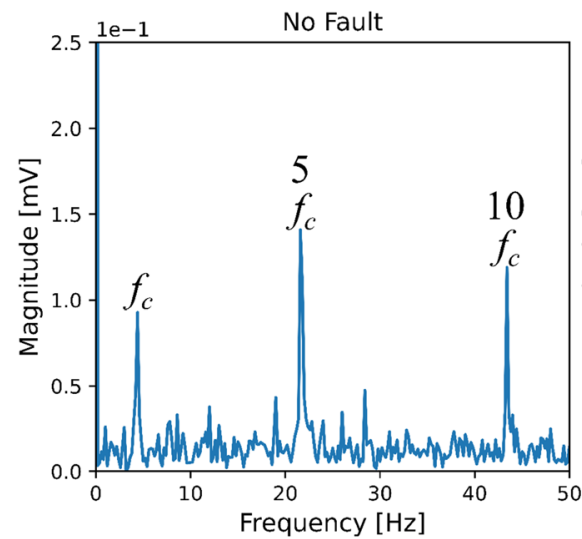

(a)

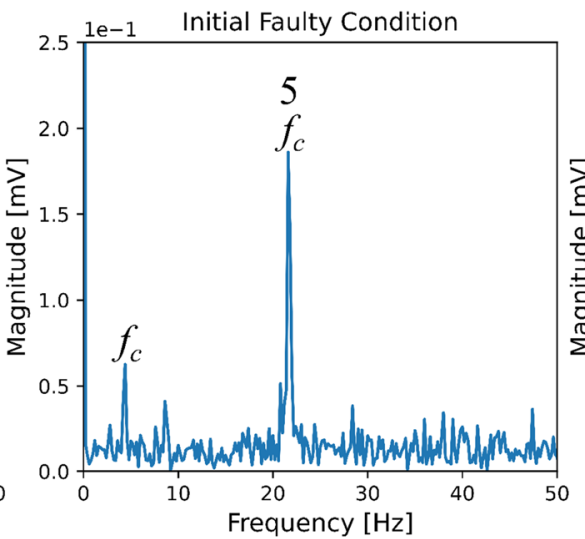

(b)

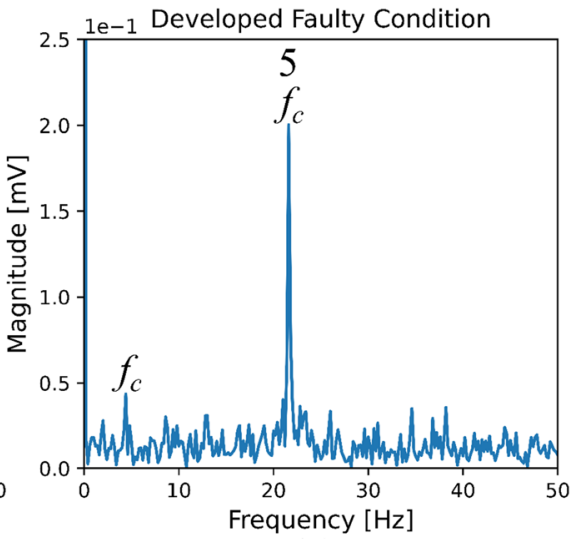

(c)

Fig. 12 Envelope spectra from the IMF-3 filtered with a $20 \mathrm{kHz}$ high-pass filter for the (a) non-faulty condition, (b) initial faulty condition and (c) developed faulty condition 


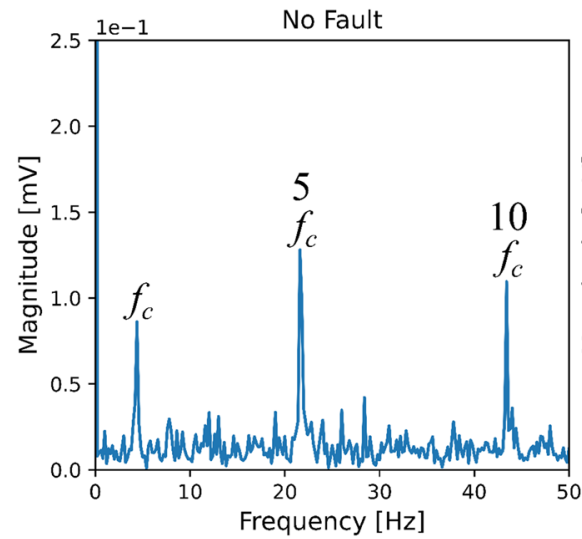

(a)

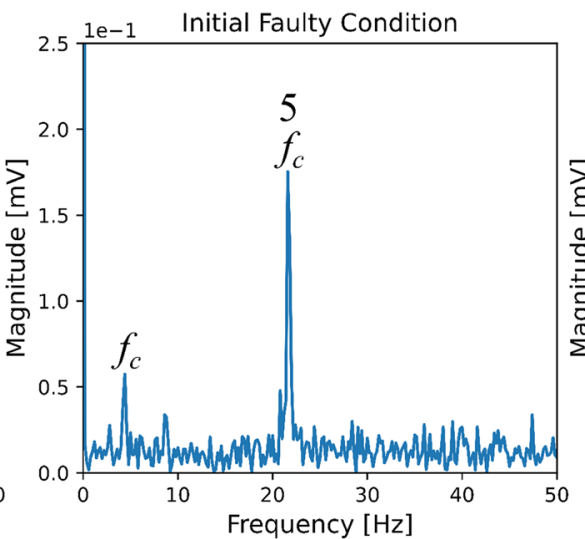

(b)

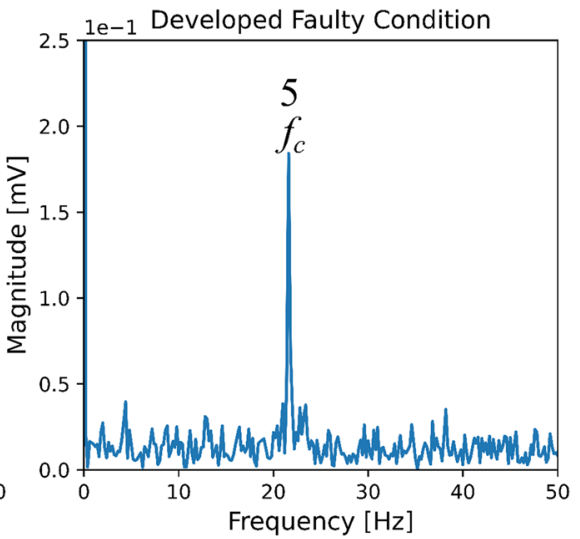

(c)

Fig. 13 Envelope spectra from the IMF-3 filtered with a 95-140 kHz band-pass filter for the a non-faulty condition, b initial faulty condition and c developed faulty condition

\section{Fast Kurtogram-Based Filtering}

In this analysis, the original signals and IMFs were filtered in the frequency band in which the signals reach a maximum kurtosis value according to the fast kurtogram. Table 1 shows the results of the fast kurtogram applied to each of the three signals considered for the averages in each case. With some exceptions, almost for each case the frequency band selected by the fast kurtogram is consistent among the three signals. The frequency band that is more repetitively selected is the band $93.75-125 \mathrm{kHz}$, except for the third IMF (all cases) and the second IMF (developed faulty condition). Regarding the kurtosis values, the maximum values occur clearly for the first IMF.

The envelope spectrum analysis was repeated for all cases considering the demodulation frequency band calculated with the fast kurtogram. The results are shown in Figs. 14, 15, 16 and 17. For cases in which the frequency band selected by the fast kurtogram was similar to the frequency band defined previosuly based on the IMF spectra $(95-140 \mathrm{kHz})$, the obtained envelope spectra result very similar as before. One particular difference occurs for the first IMF in the no faulty condition, where the more narrow frequency band selected by the fast kurtogram generates a decrease in the first harmonic $f_{c}$ (compare Figs. 9 and 15). For the second IMF (developed faulty condition) a lower frequency band was mainly selected by the fast kurtogram, which results in a more flat envelope spectrum with harmonics $f_{c}$ of low magnitude (see Fig. 16). For the third IMF a much higher frequency band was selected by the fast kurtogram, which results in envelope spectra with much noise and harmonics $f_{c}$ difficult to distinguish (see Fig. 17).
Table 1 Results of the fast kurtogram

\begin{tabular}{llllll}
\hline Condition & Signal & \multicolumn{2}{l}{ Frequency band [kHz] } & \multirow{2}{*}{ Average Kurtosis } \\
\cline { 3 - 5 } & & No. 1 & No. 2 & No. 3 & \\
\hline \multirow{2}{*}{ No fault } & Original & $93.75-125$ & $93.75-125$ & $187.5-218.8$ & 0.69 \\
& IMF-1 & $93.75-125$ & $93.75-125$ & $93.75-125$ & 660.7 \\
& IMF-2 & $93.75-125$ & $93.75-125$ & $93.75-125$ & 14.85 \\
& IMF-3 & $416.7-500$ & $375-500$ & $375-500$ & 11.69 \\
Ini. fault & Original & $93.75-125$ & $93.75-125$ & $93.75-125$ & 1.69 \\
& IMF-1 & $93.75-125$ & $93.75-125$ & $93.75-125$ & 896.2 \\
& IMF-2 & $93.75-125$ & $93.75-125$ & $93.75-125$ & 25.14 \\
& IMF-3 & $416.7-500$ & $416.7-500$ & $416.7-500$ & 14.54 \\
Dev. fault & Original & $93.75-125$ & $93.75-125$ & $93.75-125$ & 2.72 \\
& IMF-1 & $93.75-125$ & $93.75-125$ & $93.75-125$ & 610.6 \\
& IMF-2 & $62.5-93.75$ & $62.5-93.75$ & $93.75-125$ & 22.67 \\
& IMF-3 & $416.7-500$ & $416.7-500$ & $416.7-500$ & 15.15 \\
\hline
\end{tabular}




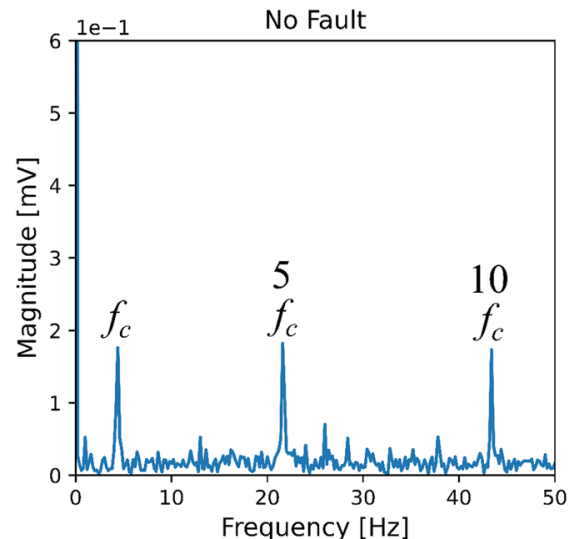

(a)

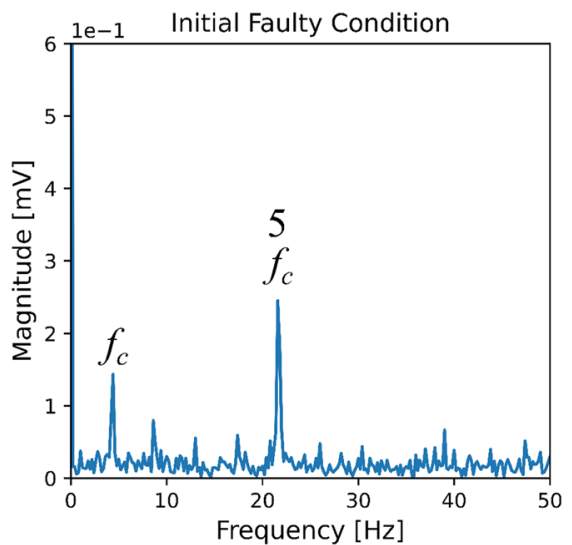

(b)

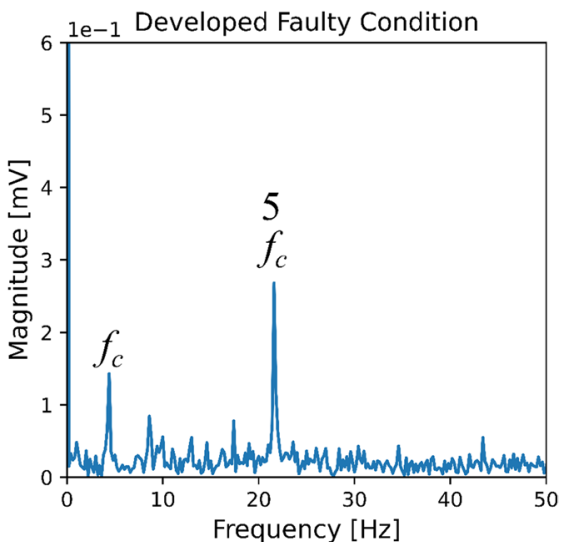

(c)

Fig. 14 Envelope spectra from the original signal filtered based on the kurtogram for the a non-faulty condition, $\mathbf{b}$ initial faulty condition and $\mathbf{c}$ developed faulty condition

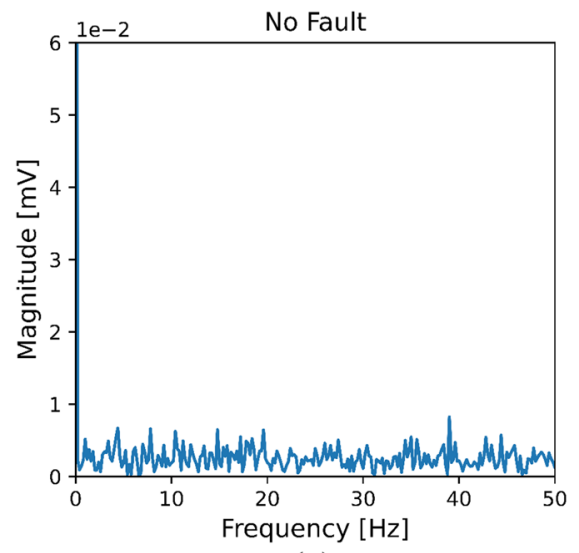

(a)

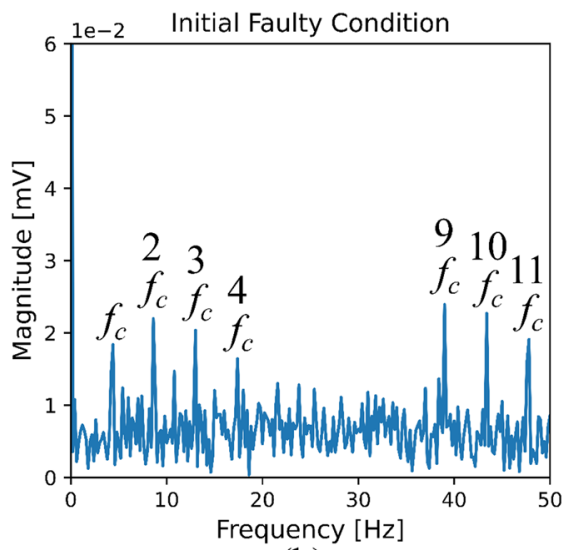

(b)

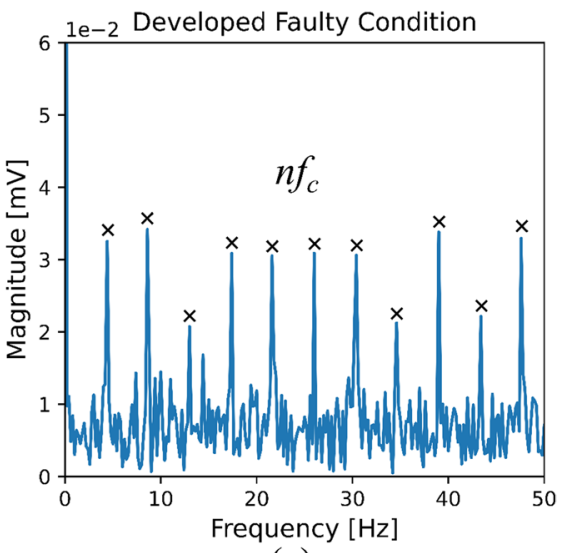

(c)

Fig. 15 Envelope spectra from the IMF-1 filtered based on the kurtogram for the a non-faulty condition, $\mathbf{b}$ initial faulty condition and $\mathbf{c}$ developed faulty condition

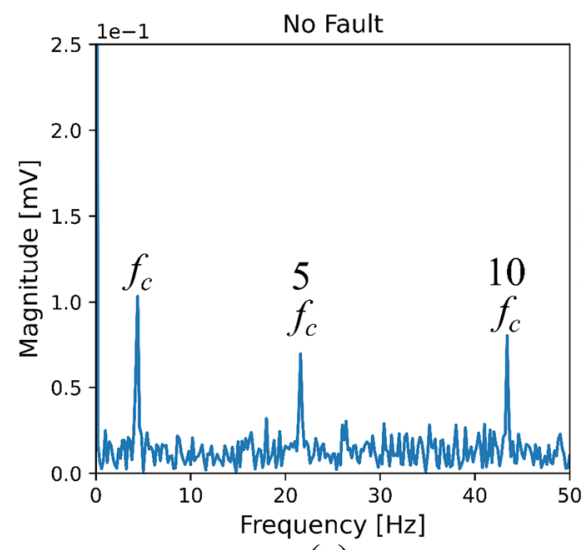

(a)

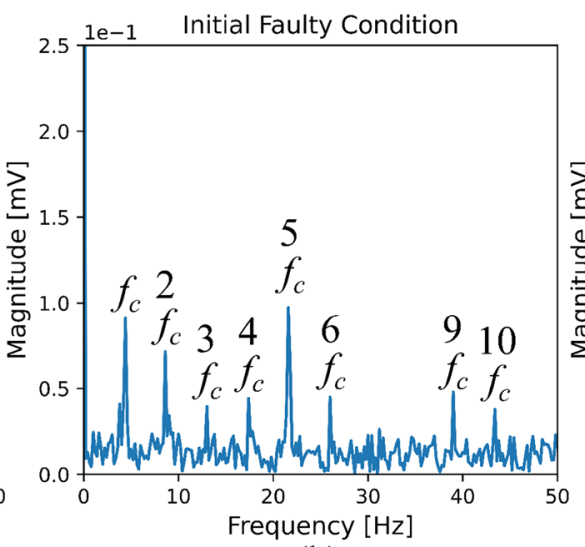

(b)

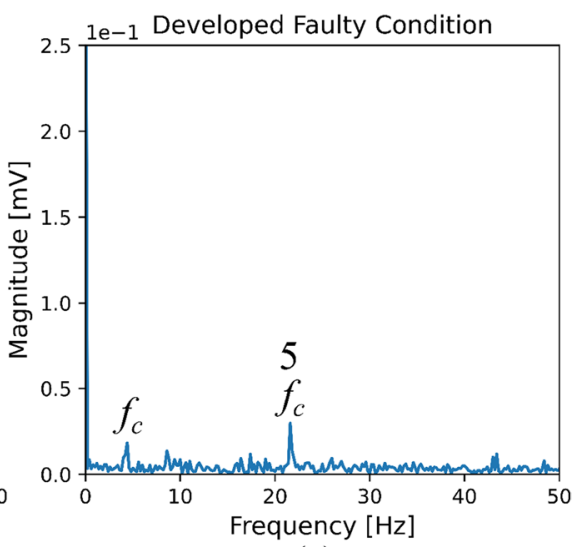

(c)

Fig. 16 Envelope spectra from the IMF-2 filtered based on the kurtogram for the a non-faulty condition, $\mathbf{b}$ initial faulty condition and $\mathbf{c}$ developed faulty condition 


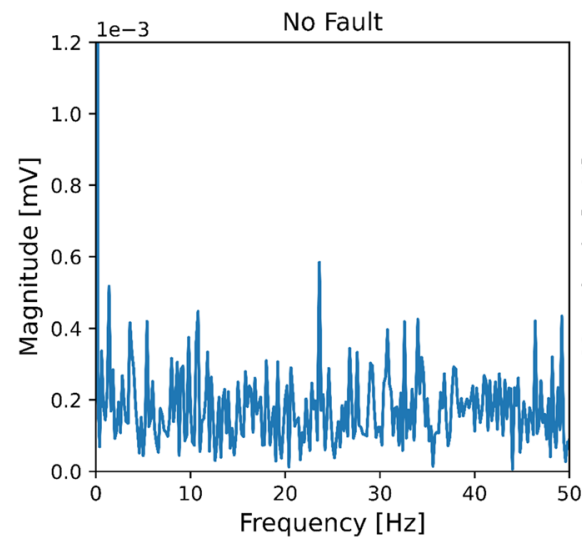

(a)

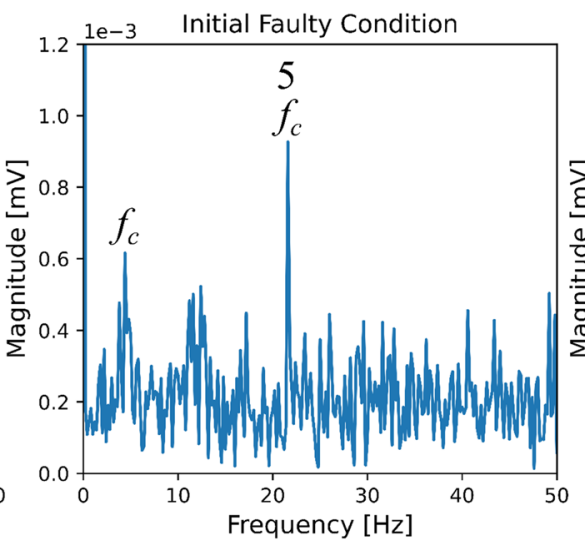

(b)

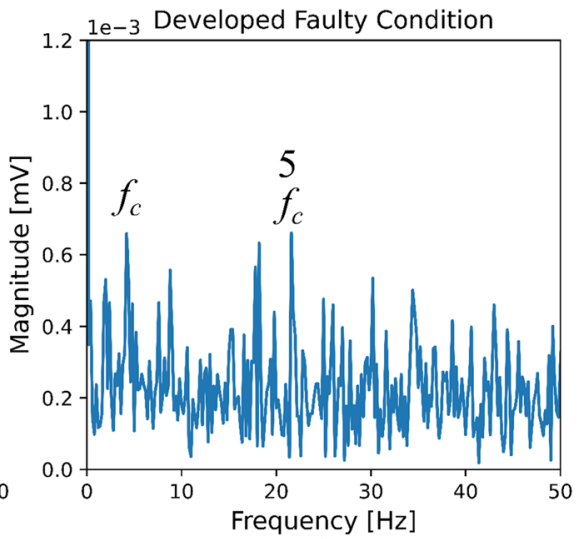

(c)

Fig. 17 Envelope spectra from the IMF-3 filtered based on the kurtogram for the a non-faulty condition, $\mathbf{b}$ initial faulty condition and $\mathbf{c}$ developed faulty condition

Table 2 MPR calculation for the cases under analysis

\begin{tabular}{llllll}
\hline Case & Signal & Demod. range & \multicolumn{2}{l}{ MPR [dB] } \\
\cline { 3 - 5 } & & & No fault & Ini. fault & Dev. fault \\
\hline 1 & Original signal & $<20 \mathrm{kHz}$ & 29.64 & 27.84 & 28.64 \\
2 & & $95-140 \mathrm{kHz}$ & 28.78 & 27.86 & 28.13 \\
3 & & Kurtogram & 29.15 & 27.90 & 28.27 \\
4 & IMF-1 & $<20 \mathrm{kHz}$ & 26.17 & 24.79 & 28.52 \\
5 & & $95-140 \mathrm{kHz}$ & 20.61 & 24.87 & 28.43 \\
6 & & Kurtogram & 22.04 & 25.19 & 28.54 \\
7 & IMF-2 & $<20 \mathrm{kHz}$ & 28.21 & 27.66 & 26.92 \\
8 & & $95-140 \mathrm{kHz}$ & 26.94 & 27.78 & 26.37 \\
9 & & Kurtogram & 26.38 & 27.89 & 26.30 \\
10 & IMF-3 & $<20 \mathrm{kHz}$ & 27.84 & 26.22 & 26.76 \\
11 & & $95-140 \mathrm{kHz}$ & 27.50 & 25.70 & 26.05 \\
12 & & Kurtogram & 19.06 & 22.54 & 22.0 \\
\hline
\end{tabular}

\section{MPR Calculation}

The MPR calculation was used to establish quantitatively the benefit of using the EMD with appropriate frequency band demodulation for fault diagnosis. Table 2 summarizes the results obtained considering the twelve presented cases. This analysis confirmed that the use of the first IMF with appropriate frequency band for demodulation was the best suited case for monitoring the fault in the ring gear (cases 5 and 6). When no fault was present, the MPR had a value of $20.61 \mathrm{~dB}$ for the fixed frequency band and a value 22.04 for the selection of the frequency band with the fast kurtogram. After that the fault was introduced, the MPR value increased to approximately $25 \mathrm{~dB}$ for both cases and after the $9.5 \mathrm{~h}$ of intermittently operation it increased to approximately $28.5 \mathrm{~dB}$ for both cases. Other cases (1, 2, 3, $4,8,9,10,11$ and 12) had an erratic behavior in their MPR values, i.e. they did not follow an increasingly tendency. For the case 7 the results show a reverse tendency in the magnitude of the harmonics, i.e. they decrease their magnitudes with fault development. For some cases $(1,2,3,4$, $7,8,10$ and 11) the MPR values were higher for the nonfaulty condition than for the initial faulty condition, which represents clearly a deficient signal processing procedure and could lead to wrong fault diagnosis. The comparison of the cases 2 and 3 (original signal) with the cases 5 and 6 (first IMF) indicated that the appropriate demodulation band does not improve the diagnostic capability on its own, but does so only in combination with the EMD.

\section{Filter Behavior of the EMD}

In this section the adaptive filtering behavior of the EMD is investigated. Considering the calculation of a particular IMF as a filtering operation, firstly the magnitude frequency responses were calculated as the frequency spectrum from a particular IMF divided by the frequency spectrum of the original AE signal. Secondly, an appropriate FIR filter was designed using least-squares error minimization to approximate the obtained magnitude frequency responses.

The results for each IMF and fault condition are shown in Fig. 18 using a number of taps 73 for the design of each FIR filter. Here the disadvantage of the mode mixing of the classical EMD can clearly be observed. Approximately, the first IMF is acting mainly as a high-pass filter over $100 \mathrm{kHz}$, the second IMF is acting mainly as a band-pass filter between 100 and $200 \mathrm{kHz}$ and the third IMF is acting mainly as a band-pass filter between 0 and $100 \mathrm{kHz}$. 


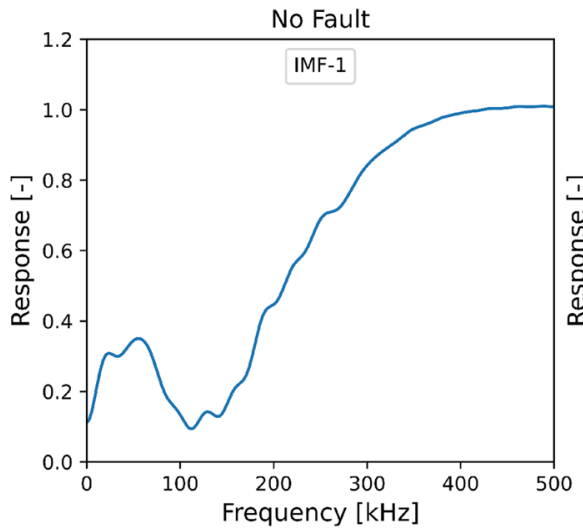

(a)

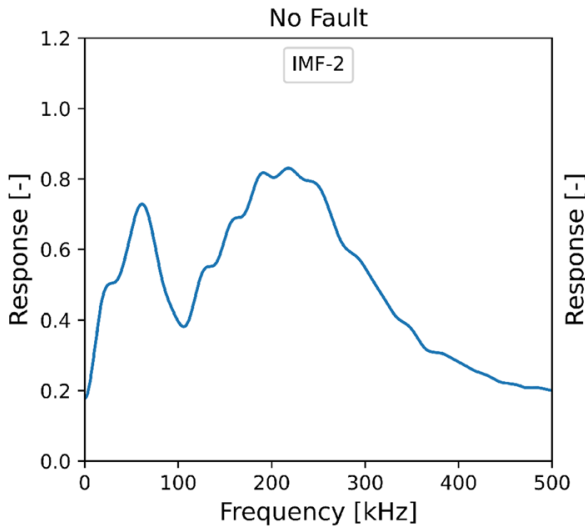

(d)

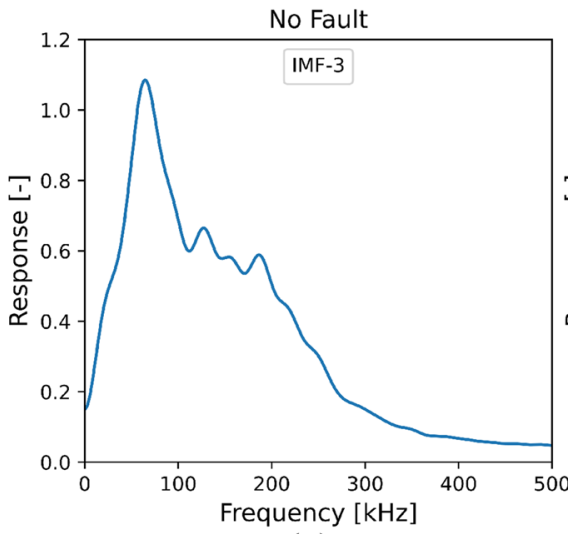

(g)

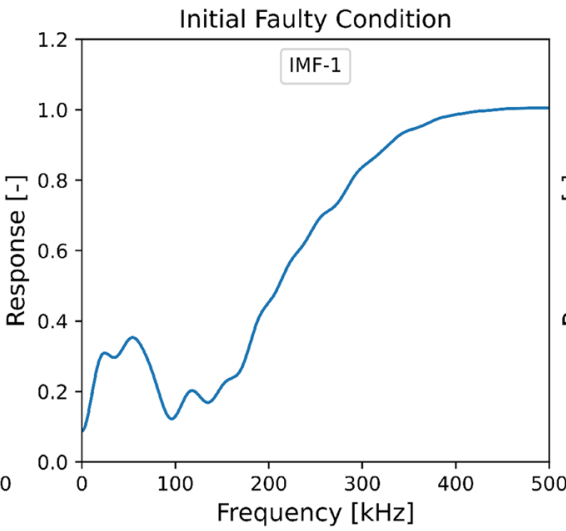

(b)

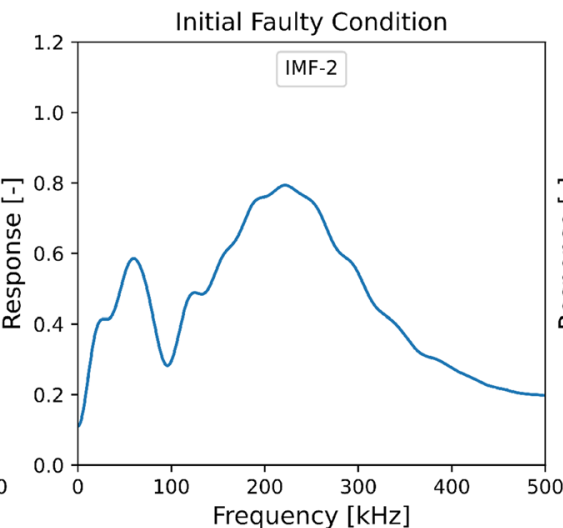

(e)

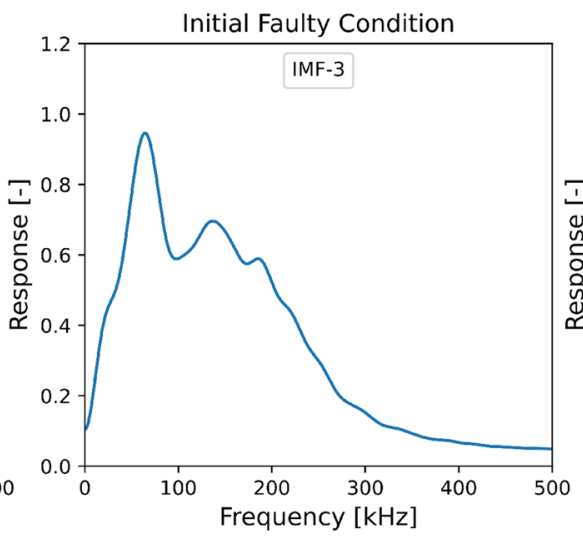

(h)

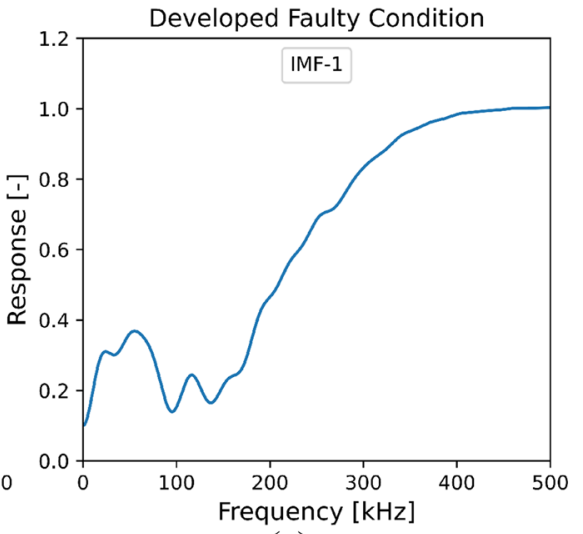

(c)

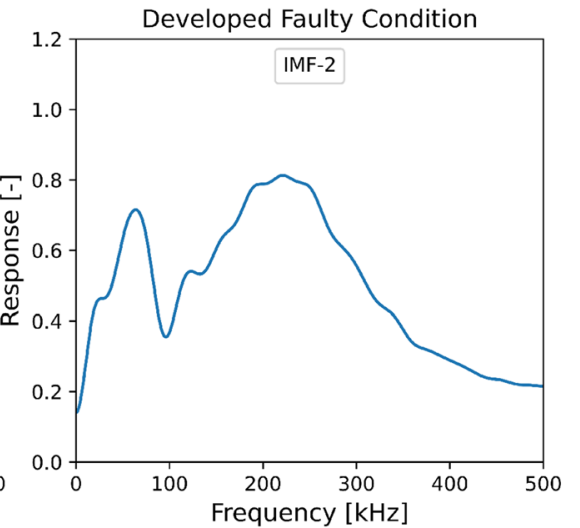

(f)

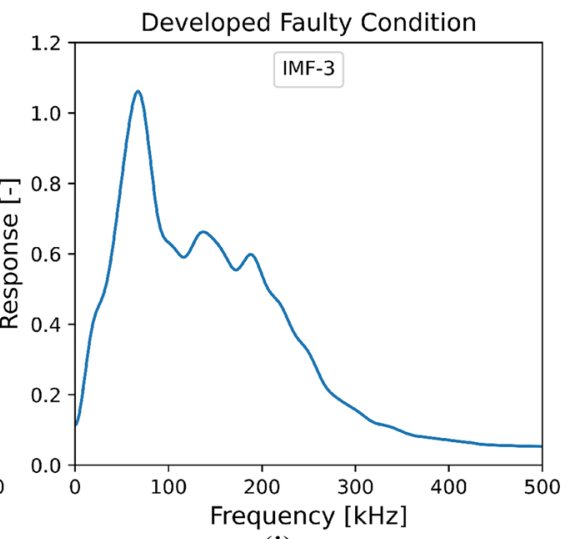

(i)
Fig. 18 Frequency responses of FIR filters calculated from: a IMF-1 without fault, b IMF-1 with initial fault, c IMF-1 with developed fault, d IMF-2 without fault, e IMF-2 with initial fault, f IMF-2 with

However, all estimated frequency responses have gain higher than zero outside these aforementioned ranges. developed fault, $\mathbf{g}$ IMF-3 without fault, h IMF-3 with initial fault and i IMF-3 with developed fault

\section{Discussion}

The obtained results show the potential of the EMD, which is a technique known for quite some time, to be used in combination with the envelope spectrum analysis to improve the gearbox diagnostics using AE signals. As demonstrated, a good correlation among fault condition and magnitude 
of fault-related components could be obtained only if the demodulation was conducted for the IMF most affected by the fault instead as for the original signal. Moreover, this correlation was even clearer if an appropriate demodulation band for the IMF is previously considered.

The calculated envelope spectra revealed that the fundamental frequency at which the planet gears interact with the ring gear is $f_{c}$ rather than $N_{p} f_{c}$. This can be due to irregular interactions or minor asymmetry among the different planet gears, which is especially relevant in the high frequencies from $\mathrm{AE}$. The envelope spectra for the original AE signals (highpass-filtered, bandpass-filtered with fixed band or bandpass-filtered based on the kurtogram) showed only minor changes among the magnitudes of the harmonics from $f_{c}$ for three fault conditions (no fault, initial fault and developed fault). This means that if no further analysis is considered, the ring gear fault could not have been detected properly. Instead, when the calculation of the envelope spectra was conducted for the first IMF (bandpass-filtered), the results revealed clear magnitude increases in the harmonics from $f_{c}$ for the faulty conditions. However, the same analysis for the second and third IMFs could not reveal major differences among the three fault conditions.

The conducted research is one of the first approaches in which envelope spectra of $\mathrm{AE}$ signals are obtained using the EMD together with appropriate demodulation band in the field of gearbox diagnostics. Within the presented case study, the selection of the suited IMF and appropriate demodulation band were conducted in two ways. The first way consisted of visual examination of the IMF spectra to find the frequency band most affected by damage progression. The second way consisted of selecting the frequency band in which the kurtosis is maximized. Here, the fast kurtogram seems as an appropriate tool for both: selection of the relevant IMF and the appropriate frequency band. However, this approach should still be confirmed with more testing since the results obtained for other high-order IMFs were not satisfactory.

Another future research topic is the application of a more advanced version of the EMD to analyze the AE signals such as the EEMD, VMD or EWT and avoid possible mode mixing as discussed from the results shown in Fig. 18. However, these methods are much more computational expensive than the EMD and the calculation time using regular hardware can become an issue for AE signals measured at high frequency ranges over $1 \mathrm{MHz}$. In this study, this calculation time was in the range of several hours per second of $\mathrm{AE}$ signal.

Despite the good results, the use of AE measurements for gearbox diagnostics has also some shortcomings. Firstly, the AE sensor must be located as near as possible to the component under consideration, since AE waves are highly attenuated by surface interfaces. This is not the case of vibration sensors, which are normally located on a near bearing and are much less affected by surface interfaces. Secondly, the AE measured on gearboxes is highly affected by operating conditions such as rotational speed and lubricant temperature, and less affected by the load depending on the lubrication regime as indicated by Tan and Mba [31]. Thirdly, because of the high sampling rate required for their measurement, the analysis of AE signals can be time-consuming and its storage can become problematic.

\section{Conclusions}

AE signals were measured in a PG test bench under three different damage conditions: non-faulty condition, initial faulty condition with a seeded fault in a tooth from the ring gear and a developed faulty condition after $9.5 \mathrm{~h}$ of intermittent operation. The envelope spectrum was used as analysis method to distinguish among the three conditions. The use of the EMD as a complementary signal processing technique was investigated. The following conclusions can be deduced from the presented results:

1. Without appropriate signal processing, the analysis of envelope spectra from AE signals could not be able to clearly diagnose the localized fault in the ring gear.

2. The frequency content of the IMFs obtained by the EMD algorithm has the potential to reveal frequency bands in which damage progression takes place.

3. Selecting the appropriate IMF and demodulation frequency band can effectively reveal spectral components related to faults.

4. The fast kurtogram technique seems to be suitable tool for IMF and frequency band selection. Although this approach is usual for vibration signals, it should be confirmed with more testing for the case of AE signals.

5. The performance of the envelope spectrum analysis for gearbox diagnostics using AE signals can be considerably improved by the use of the EMD as complementary signal processing technique.

There is still limited research on the use of techniques such as EMD for gearbox diagnostics based on AE signals. This paper contributes to provide evidence of the potential of the EMD in combination with the envelope spectrum analysis of AE signals. However, in account of the drawbacks from the original EMD, further research shall focus on avoiding mode mixing, automation of the IMF and demodulation band selection and optimizing the computations of the sifting process for AE data. 
Funding Open Access funding enabled and organized by Projekt DEAL.

\section{Declarations}

Conflict of interest On behalf of all authors, the corresponding author states that there is no conflict of interest.

Open Access This article is licensed under a Creative Commons Attribution 4.0 International License, which permits use, sharing, adaptation, distribution and reproduction in any medium or format, as long as you give appropriate credit to the original author(s) and the source, provide a link to the Creative Commons licence, and indicate if changes were made. The images or other third party material in this article are included in the article's Creative Commons licence, unless indicated otherwise in a credit line to the material. If material is not included in the article's Creative Commons licence and your intended use is not permitted by statutory regulation or exceeds the permitted use, you will need to obtain permission directly from the copyright holder. To view a copy of this licence, visit http://creativecommons.org/licenses/by/4.0/.

\section{References}

1. Antoni J (2006) The spectral kurtosis: a useful tool for characterising non-stationary signals. Mech Syst Signal Process 20(2):282 307. https://doi.org/10.1016/j.ymssp.2004.09.001

2. Antoni J (2006) Fast computation of the kurtogram for the detection of transient faults. Mech Syst Signal Process 21(1):108-124. https://doi.org/10.1016/j.ymssp.2005.12.002

3. Antoni J (2007) Fast computation of the kurtogram for the detection of transient faults. Mech Syst Signal Process 21(1):108-124. https://doi.org/10.1016/j.ymssp.2005.12.002

4. Antoni J, Randall RB (2006) The spectral kurtosis: application to the vibratory surveillance and diagnostics of rotating machines. Mech Syst Signal Process 20(2006):308-331. https://doi.org/10. 1016/j.ymssp.2004.09.002

5. Chen H, Chen P, Chen W et al (2017) Wind turbine gearbox fault diagnosis based on improved EEMD and Hilbert square demodulation. Appl Sci 7(2):128. https://doi.org/10.3390/app7020128

6. Chen J, Pan J, Li Z, Zi Y, Chen X (2016) Generator bearing fault diagnosis for wind turbine via empirical wavelet transform using measured vibration signals. Renew Energy 89(2016):80-92. https://doi.org/10.1016/j.renene.2015.12.010

7. Dragomiretskiy K, Zosso D (2014) Variational mode decomposition. IEEE Trans Signal Process 62(3):531-544. https://doi.org/ 10.1109/TSP.2013.2288675

8. Eftekharnejad B, Mba D (2009) Seeded fault detection on helical gears with acoustic emission. Appl Acoust 70(2009):547-555. https://doi.org/10.1016/j.apacoust.2008.07.006.nejad

9. Feng Z, Liang M, Zhang Y et al (2012) Fault diagnosis for wind turbine planetary gearboxes via demodulation analysis based on ensemble empirical mode decomposition and energy separation. Renew Energy 47(2012):112-126. https://doi.org/10.1016/j. renene.2012.04.019

10. Feng Z, Zhang D, Zuo MJ (2017) Planetary gearbox fault diagnosis via joint amplitude and frequency demodulation analysis based on variational mode decomposition. Appl Sci 7(8):775. https:// doi.org/10.3390/app7080775

11. Flandrin P, Rilling G, Goncalves P (2004) Empirical mode decomposition as a filter bank. IEEE Signal Proc Lett 11(2):112-114. https://doi.org/10.1109/LSP.2003.821662
12. Gao L, Zai F, Su S et al (2011) Study and application of acoustic emission testing in fault diagnosis of heavy duty gears. Sensors 11(1):599-611. https://doi.org/10.3390/s110100599

13. Hemmati F, Alqaradawi M, Gadala M (2016) Rolling element bearing fault diagnostics using acoustic emission technique and advanced signal processing. Proc Inst Mech Eng Part J: J Eng 230(1):64-77. https://doi.org/10.1177/1350650115591233

14. Huang M, Jiang L, Liaw P et al (1998) Using acoustic emission in fatigue and fracture materials research. JOM-e: Res Summ 50(11):1-12

15. Huang N, Shen Z, Long S et al (1998) The empirical mode decomposition and the Hilbert spectrum for nonlinear and non-stationary time series analysis. Proc R Soc A: Math Phys 454(1998):903995. https://doi.org/10.1098/rspa.1998.0193

16. Kannan V, Li H, Dao DV (2019) Demodulation band optimization in envelope analysis for fault diagnosis of rolling element bearings using a real-coded genetic algorithm. IEEE Access 7(2019):168828-168838. https://doi.org/10.1109/ACCESS.2019. 2954704

17. Kedadouche M, Thomas M, Tahan A (2014) Empirical mode decomposition of acoustic emission for early detection of bearing defects. In: Dalpiaz G et al. (eds) Advances in Condition Monitoring of Machinery in Non-Stationary Operations. Lecture Notes in Mechanical Engineering. Springer, Berlin, Heidelberg. https://doi. org/10.1007/978-3-642-39348-8-31

18. Leaman F, Vicuña CM, Clausen E (2021) Comparative case studies on ring gear fault diagnosis of planetary gearboxes using vibrations and acoustic emissions. Forsch Ingenieurwes. https:// doi.org/10.1007/s10010-021-00451-4

19. Li Z, Chen J, Zi Y et al (2017) Independence-oriented VMD to identify fault feature for wheel set bearing fault diagnosis of high speed locomotive. Mech Syst Signal Process 85(2017):512-529. https://doi.org/10.1016/j.ymssp.2016.08. 042

20. Li R, He D (2012) Rotational machine health monitoring and fault detection using EMD-based acoustic emission feature quantification. IEEE Trans Instrum Meas 61(4):990-1001. https://doi.org/ 10.1109/TIM.2011.2179819

21. Liu H, Huang W, Wang S, Zhu Z (2014) Adaptive spectral kurtosis filtering based on Morlet wavelet and its application for signal transients detection. Signal Process 96A(2014):118-124. https:// doi.org/10.1016/j.sigpro.2013.05.013

22. Liu B, Riemenschneider S, Xu Y (2006) Gearbox fault diagnosis using empirical mode decomposition and Hilbert spectrum. Mech Syst Signal Process 20(2006):718-734. https://doi.org/10.1016/j. ymssp.2005.02.003

23. Loutas TH, Sotiriades G, Kalaitzoglou I et al (2009) Condition monitoring of a single-stage gearbox with artificially induced gear cracks utilizing on-line vibration and acoustic emission measurements. Appl Acoust 70(2009):1148-1159. https://doi.org/10. 1016/j.apacoust.2009.04.007

24. Loutridis SJ (2004) Damage detection in gear systems using empirical mode decomposition. Eng Struct 26(2004):1833-1841. https://doi.org/10.1016/j.engstruct.2004.07.007

25. McDonald GL, Zhao Q, Zuo MJ (2012) Maximum correlated kurtosis deconvolution and application on gear tooth chip fault detection. Mech Syst Signal Process 33(2012):237-255. https:// doi.org/10.1016/j.ymssp.2012.06.010

26. Novoa AB, Vicuña CM (2016) New aspects concerning the generation of acoustic emissions in spur gears, the influence of operating conditions and gear defects in planetary gearboxes. Insight 58(1):18-27. https://doi.org/10.1784/insi.2016.58.1.18

27. Potamianos A, Maragos P (1994) A comparison of the energy operator and the Hilbert transform approach to signal and speech demodulation. Signal Process 37(1):95-120. https://doi.org/10. 1016/0165-1684(94)90169-4 
28. Sawalhi N, Randall RB, Endo H (2007) The enhancement of fault detection and diagnosis in rolling element bearings using minimum entropy deconvolution combined with spectral kurtosis. Mech Syst Signal Process 21(6):2616-2633. https://doi.org/10. 1016/j.ymssp.2006.12.002

29. Schroeder MR (2013) Computer speech: recognition, compression, synthesis, vol 35. Springer Science \& Business Media, Berlin, p 2013

30. Tan CK, Irving P, Mba D (2007) A comparative experimental study on the diagnostic and prognostic capabilities of acoustics emission, vibration and spectrometric oil analysis for spur gears. Mech Syst Signal Process 21(1):208-233. https://doi.org/10. 1016/j.ymssp.2005.09.015

31. Tan CK, Mba D (2005) Identification of the acoustic emission source during a comparative study on diagnosis of a spur gearbox. Tribol Int 38(5):469-480. https://doi.org/10.1016/j.triboint.2004. 10.007

32. Teng W, Wang F, Zhang K et al (2014) Pitting fault detection of a wind turbine gearbox using empirical mode decomposition. $\mathrm{J}$ Mech Eng 60(2014):12-20. https://doi.org/10.5545/sv-jme.2013. 1295

33. Wang L, Shao Y, Cao Z (2018) Optimal demodulation subband selection for sun gear crack fault diagnosis in planetary gearbox. Measurement 125(2018):554-563. https://doi.org/10.1016/j. measurement.2018.05.023
34. Wu Z, Huang N (2009) Ensemble empirical mode decomposition: a noise-assisted data analysis method. Adv Adapt Data Anal 1(2009):1-41. https://doi.org/10.1142/S1793536909000047

35. Yoon J, He D (2012) planetary gearbox fault diagnostic method using acoustic emission sensors. IET Sci Meas Technol 9(8):936944. https://doi.org/10.1049/iet-smt.2014.0375

36. Yu D, Wang M, Cheng X (2016) A method for the compound fault diagnosis of gearboxes based on morphological component analysis. Measurement 91(2016):519-531. https://doi.org/10. 1016/j.measurement.2016.05.087

37. Zhang X, Kang J, Xiao L et al (2015) A new improved kurtogram and its application to bearing fault diagnosis. Shock Vib 2015(2015):385412. https://doi.org/10.1155/2015/385412

38. Zhang C, Wang Y, Deng W (2020) Fault diagnosis for rolling bearings using optimized variational mode decomposition and resonance demodulation. Entropy 22(7):739. https://doi.org/10. $3390 / \mathrm{e} 22070739$

Publisher's Note Springer Nature remains neutral with regard to jurisdictional claims in published maps and institutional affiliations. 